\title{
IFI and ISI Premitigation for Block-Code-Modulated Noncoherent UWB Impulse Radio: A Code Optimization Approach
}

\author{
Hui Gao, Student Member, IEEE, Xin Su, Member, IEEE, Tiejun Lv, Member, IEEE, \\ Shaoshi Yang, Student Member, IEEE, and Yueming Lu
}

\begin{abstract}
Codeword matching and signal aggregation (CMSA) is a recently proposed low-complexity noncoherent receiver for block-code-modulated ultrawideband impulse radio (UWB-IR) systems. As the frame/symbol duration is shortened to boost data rate, interframe interference (IFI) or intersymbol interference (ISI) occurs and degrades the detection performance of CMSA. In this paper, an effective IFI/ISI premitigation scheme is proposed for CMSA through a code optimization approach. By employing a tailored interference model that highlights the codeword properties, the system performance in the presence of moderate IFI/ISI is evaluated, and the average collected channel gain is introduced as the metric for code optimization. With the primary focus on binary modulation, the following two IFI/ISI-robust code properties are generalized: 1) shifted orthogonality and 2) shifted repetition. Based on these properties, the optimal code is constructed. It is observed that, when the optimal code occurs, the leaked signal energy or the interference can partially be used to enhance the detection performance of CMSA in the presence of IFI/ISI. Unlike most of the existing IFI/ISI mitigation schemes for noncoherent UWB-IR, which mainly focus on signal processing after the nonlinear detector, the optimized code is exploited to aggregate the leaked signal energy, along with the linear predetection operation already involved in the CMSA receiver. Both analysis and simulation show that a distinct performance improvement is achieved.
\end{abstract}

Index Terms-Block code (BC) modulation, interframe interference (IFI), intersymbol interference (ISI), noncoherent, premitigation, ultrawideband impulse radio (UWB-IR).

Manuscript received June 22, 2011; revised November 16, 2011; accepted January 24, 2012. Date of publication February 14, 2012; date of current version May 9, 2012. This work was supported in part by the National Natural Science Foundation of China under Grant 60972075, the Important National Science and Technology Specific Projects of China under Grant 2011ZX03003002-01, and the National 973 Program of China under Grant 2011CB302702. This paper was presented in part at the 2010 IEEE International Conference on Communications Cape Town, South Africa. The review of this paper was coordinated by Dr. X. Dong.

H. Gao and T. Lv are with the School of Information and Communication Engineering, Beijing University of Posts and Telecommunications, Beijing 100876, China (e-mail: huigao@bupt.edu.cn; lvtiejun@bupt.edu.cn).

$\mathrm{X}$. Su is with Research Institute of Information Technology of Tsinghua University, Beijing 100084, China (e-mail: suxin@ tsinghua.edu.cn).

$\mathrm{S}$. Yang is with the School of Electronics and Computer Science, University of Southampton, SO17 1BJ Southampton, U.K. (e-mail: sy7g09@ecs. soton.ac.uk).

Y. Lu is with the Key Laboratory of Trustworthy Distributed Computing and Service, Ministry of Education, Beijing University of Posts and Telecommunications, Beijing 100876, China.

Digital Object Identifier 10.1109/TVT.2012.2187938

\section{INTRODUCTION}

$\mathbf{U}$ LTRAWIDEBAND impulse radio (UWB-IR) communication has widely been considered to be a promising solution for wireless personal area networks and wireless sensor networks due to its unique potential for achieving high data rate at low cost [1], [2]. In particular, UWB-IR exploits very narrow pulses of subnanoseconds to transmit information, and the received signal consists of a large number of resolvable multipath components (MPCs) after passing through the ultrawideband (UWB) channel. The Rake receiver is designed to coherently combine these MPCs [3]. However, the UWB channel is characterized by dense multipath, and the stringent requirements on channel estimation and synchronization make it difficult and costly to implement the optimal coherent receiver for UWB-IR. To avoid the complicated treatments on the UWB channel, noncoherent UWB-IR systems are proposed, with the good performance-complexity tradeoff [4]-[11]. The conventional transmitted-reference (TR) [4] scheme, the differential transmitted-reference [5] scheme, and their performanceenhancing variants, the averaged transmitted-reference (ATR) [6] and the frame-level differential transmitted-reference (FDTR) schemes [7], were considered the main solutions for noncoherent UWB-IR systems. However, these conceptually simple TR schemes [4]-[7] may require wideband analog delay line, which is often tens of nanoseconds long for UWB channels. Noting the difficulty of realizing such a long analog delay line in an integrated fashion, some novel TR schemes have been proposed to shorten or even bypass the analog delay line, such as the transmitted-reference pulse cluster [8], the frequency-shift reference [9], and the code-multiplexed transmitted-reference (CMTR) [10] schemes. Recently, a block code (BC)-modulated noncoherent UWB-IR scheme has been proposed with the codeword matching and signal aggregation (CMSA) receiver [11]. CMSA combines the linear filtering (with respect to the trial codewords) and the energy detection to make the final decision variable. It is also pointed out in [11] that BC-CMSA outperforms both the ATR [6] and FDTR [7] schemes in terms of the bit error rate (BER). Although BC-CMSA may still require an analog receiver with delay lines or a sophisticated digital receiver for implementation, the scheme itself is of interest, and its potential for high data rate has yet to be explored from the theoretical perspective first, which is the focus of this paper. 
For many application scenarios, transmission with higher data rate is required, which usually leads to shortened frame/symbol duration for UWB-IR. However, the maximum excess delay of the UWB channel [12] limits the improvement of the data rate: when the frame/symbol duration is shortened within the channel maximum excess delay, the interframe interference (IFI) and intersymbol interference (ISI) occur and often degrade detection performance. Combating against IFI/ISI is crucial. Hence, it is challenging to fulfill the UWB-IR's goal of high-data-rate transmission with a relatively simple noncoherent receiver. In addition, the existing noncoherent UWB-IR schemes often employ the autocorrelation receiver (AcR) or the energy detector (ED) at the back end of the receivers [13], and both of them involve nonlinear operations that, in the presence of IFI/ISI, generate a nonlinear interference component in the decision variable. Therefore, classical linear IFI/ISI mitigation methods, such as linear equalizations in [14], cannot readily be implemented into the back end of the receiver. A number of algorithms have recently been developed to address the IFI/ISI mitigation problem for noncoherent UWB-IR. These algorithms mainly focus on equalization [7], [15], [16] and multiple-symbol detection (MSD) or maximumlikelihood sequence estimation (MLSE) [7], [17], [18]. More specifically, an ED-based decision feedback equalizer is proposed in [15] for the on/off-keying-modulated UWB-IR. In comparison, the second-order Volterra system is introduced in [7] for the FDTR system, which spurs the works on the adaptive inverse-modeling equalizer [7], the nonlinear minimum mean square error (MMSE) equalizer [16], for the mitigation of IFI/ISI. However, all of these equalization schemes require the estimation of the equalizer's coefficients, which are channel dependent; therefore, training overheads are inevitable, and costly efforts are needed to ensure estimation accuracy. Aside from equalization, MSD or MLSE is proposed in [7] with full channel-state information (CSI) and in [17] with partial CSI. A multiple-symbol differential detection scheme is derived in [18] based on the generalized-likelihood ratio test approach [19]. No more training or estimation for CSI is needed in [18]; however, the Viterbi algorithm that was used for detection still increases the complexity of the receiver. In summary, the aforementioned equalization and MSD or MLSE schemes either need CSI estimation or heavily rely on signal processing after the nonlinear detector at the back end of the receiver, which inevitably results in relatively high implementation complexity at the receiver. Therefore, the question follows: Is it possible to combat the IFI/ISI before the nonlinear detector by using simple but effective methods?

In this paper, an effective IFI/ISI premitigation scheme is proposed for the ED-based BC-CMSA system through a code optimization approach. By exploiting the special properties of the optimized $\mathrm{BC}$, the proposed scheme aims at mitigating the moderate IFI/ISI of BC-CMSA along with the simple linear filtering operation involved in CMSA but does not attempt to mitigate IFI/ISI after the nonlinear ED. Benefitting from this approach, the potential of the BC-CMSA system for improving the data rate is fulfilled without increasing the complexity of the receiver. Note that the conjugate code pair that was obtained from the Walsh-Hadamard matrix was used to optimize the performance of the CMTR system in the presence of IFI [10], and an interesting design of delay-hopping code and chip code was proposed in [20] to suppress the moderate IFI/ISI for the AcR-based FDTR system. In contrast to [10] and [20], our scheme is dedicated to the BC-CMSA system and is developed in a different framework. A brief demonstration of the approach was presented in a conference version of this paper [21]; however, the influence of the adopted $\mathrm{BC}$ on the performance of the BC-CMSA system in the presence of IFI/ISI was not quantified, and the optimality of the optimized BC was not proved. This paper extends the development in [21] and makes the main contributions as follows.

1) We investigate the performance of BC-CMSA in the presence of moderate IFI/ISI and reveal the intrinsic connection between the adopted $\mathrm{BC}$ and the overall system performance. In particular, we introduce an interference model, called the layered-interference (LI) model, to highlight codeword behaviors of the BC-CMSA system in the presence of IFI/ISI. Based on the adjacent interference pattern (AIP) exhibited in the LI model, we show the connection between the adopted $\mathrm{BC}$ and the system performance by a BC-dependent average collected channel gain (CCG), which serves as a practical performance metric and inspires the code optimization. Although the system performance of the interference-free BC-CMSA system has been analyzed in [22], the extension to the $\mathrm{BC}-\mathrm{CMSA}$ system in the presence of IFI/ISI has not been studied.

2) With the primary focus on binary modulation, we first derive two desired code properties, called shifted orthogonality and shifted repetition, for the IFI/ISI-robust BC-CMSA system. The chip code that was designed in [20] is based on the orthogonality of an ordinary orthogonal BC, e.g., the Walsh-Hadamard BC, whereas the proposed two special code properties has not been exploited. Furthermore, it is demonstrated that the union of the two properties enables an optimized BC to maximize the average CCG in the presence of moderate IFI/ISI and, therefore, improve the robustness of the BC-CMSA system to IFI/ISI.

3) Constrained by the two properties, we construct the optimal BC for the BC-CMSA system with binary modulation, and we further prove its optimality in terms of the average CCG. Interestingly, the optimal BC shapes the AIPs to fit the CMSA operation: the leaked energy of the previous frame/symbol, which is considered the interference, is partially exploited to increase the energy that was aggregated for the desired symbol before the ED but makes little contribution to the undesired symbol. This way, the average CCG is maximized, and premitigation on IFI/ISI is achieved by a simple linear filtering operation involved in the CMSA receiver.

The rest of this paper is organized as follows. The system model is described in Section II. In Section III, based on the LI model, the performance of BC-CMSA in the presence of IFI/ISI is analyzed, and the average CCG is introduced to guide the optimal BC construction. The optimal BC is construed in Section IV to bring forth the premitigation of IFI/ISI. 
TABLE I

LIST OF THE KEY VARIABLES

\begin{tabular}{|c|c|}
\hline Variable/Notation & Definition \\
\hline$N_{f}$ & $\begin{array}{l}\text { Number of pulses/frames to transmit one sym- } \\
\text { bol/codeword }\end{array}$ \\
\hline$T_{f}$ & Duration of one frame \\
\hline $\mathbf{B}_{2 \times N_{f}}$ & Binary codebook in terms of a $2 \times N_{f}$ matrix \\
\hline $\mathbf{b}^{(m)^{\prime}}$ & $\begin{array}{l}\text { Codeword in terms of the } m^{t h} \text { row vector of } \\
\mathbf{B}_{2 \times N_{f}}, m=1,2\end{array}$ \\
\hline $\mathbf{b}_{i}$ & The $i^{t h}$ transmitted codeword \\
\hline$m_{i}$ & $\begin{array}{l}\text { Codeword index of } \mathbf{b}_{i} \text {, i.e., } \mathbf{b}_{i}=\mathbf{b}^{\left(m_{i}\right)}, m_{i}= \\
1,2, \forall i\end{array}$ \\
\hline$d=\left(m_{i-1}, m_{i}\right)$ & $\begin{array}{l}\text { Adjacent interference pattern, } d \in\{1,2\} \times \\
\{1,2\}\end{array}$ \\
\hline$\tilde{\mathbf{b}}_{i, p}(d)$ & $\begin{array}{l}\text { The } p \text {-frame shifted version of } \mathbf{b}_{i}, p= \\
0,1, \ldots, N_{f}-1\end{array}$ \\
\hline$c_{i, p}^{(m)}(d)$ & $\begin{array}{l}\text { Codeword correlation between } \mathbf{b}^{(m)} \text { and } \\
\tilde{\mathbf{b}}_{i, p}(d)\end{array}$ \\
\hline $\mathbf{c}_{i}^{(m)}(d)$ & $\begin{array}{l}\text { Row vector containing element } c_{i, p}^{(m)}(d), p= \\
0,1, \ldots, N_{f}-1\end{array}$ \\
\hline$r_{k}(t)$ & $\begin{array}{l}\text { The } k^{t h} \text { frame-long waveform of the received } \\
\text { signal }\end{array}$ \\
\hline $\mathbf{r}_{i}(t)$ & $\begin{array}{l}\text { Waveform vector of the } i^{t h} \text { symbol-long re- } \\
\text { ceived signal }\end{array}$ \\
\hline$g(t)$ & Overall channel response \\
\hline$T_{g}$ & Duration of $g(t)$ \\
\hline$g_{p}(t)$ & $\begin{array}{l}\text { The } p^{t h} \text { partial channel response of } g(t), p= \\
0,1, \ldots,\left[T_{g} / T_{f}\right]-1\end{array}$ \\
\hline $\bar{\varepsilon}_{g}$ & Average power of $g(t)$ \\
\hline $\bar{\varepsilon}_{p}$ & Average power of $g_{p}(t), p=0,1, \ldots, N_{f}-1$ \\
\hline$\lambda$ & Ratio between $\bar{\varepsilon}_{0}$ and $\bar{\varepsilon}_{g}$ \\
\hline$\gamma_{i}(d)$ & $\begin{array}{l}\text { Average receive SNR of the } i^{\text {th }} \text { symbol condi- } \\
\text { tioned on } d\end{array}$ \\
\hline $\begin{array}{l}\beta_{i}(d) \\
\beta_{B C}\end{array}$ & $\begin{array}{l}\text { Collected channel gain conditioned on } d \\
\text { Average collected channel gain of particular } \\
\text { block code B }\end{array}$ \\
\hline
\end{tabular}

Simulation results and discussions are given in Section V. Finally, Section VI concludes this paper. Table I summarizes some of the key variables used in this paper.

Notation: Boldface letters denote the vector or matrix. $\mathbf{A}_{M \times N}$ represents an $M \times N$ matrix, and $[\mathbf{A}]_{m, n}$ is the $(m, n)$-th entry of matrix $\mathbf{A} \cdot \mathbf{A}^{T}$ is the transpose of $\mathbf{A} \cdot \mathbf{A}=$ $\operatorname{diag}\left\{a_{0}, a_{1}, \ldots, a_{N-1}\right\}$ is an $N \times N$ diagonal matrix. The symbols $*$ and $\otimes$ stand for convolution and Kronecker product operations, respectively. $\lceil\cdot\rceil$ and $\lfloor\cdot\rfloor$ stand for the integer ceil and floor operation, respectively. $\bmod _{N}[x]$ takes the modulus after division as $\bmod _{N}[x]=x-\lfloor x / N\rfloor \times N . C_{n}^{m}$ is the number of $n$-combinations from a given set of $m$ elements.

\section{SySTEM MODEL}

$\mathrm{BC}$ modulation transmits information by codeword that is chosen from a codebook. In this paper, we focus on binary modulation, which achieves a good complexity-performance tradeoff for the BC-CMSA system. ${ }^{1}$ The code matrix is $\mathbf{B}_{2 \times N_{f}}$

\footnotetext{
${ }^{1}$ BC-CMSA can achieve $M$-ary $(M>2)$ modulation by using a codebook $\mathbf{B}_{M \times N_{f}}$ and a more complicated receiver [11]. In this scenario, one codeword with length $N_{f}$ can carry a maximum of $\log _{2} N_{f}$ bits of information, or equivalently, the minimum frame number that is required for $M$-ary modulation is $N_{f}=M$. By defining the transmission efficiency as $\eta:=\log _{2} M / M$, we can observe that, when $M=2,4, \eta=\eta_{\max }=0.5$, and when $M>4$, $\eta$ monotonically decreases with $M$. In addition, $M$ detection branches are needed for $M$-ary modulation in the BC-CMSA system. For high-data-rate transmission, we jointly consider the transmission efficiency and receiver complexity to choose binary modulation as the candidate.
}

with each row vector $\mathbf{b}^{(m)}, m=1,2$ serving as a codeword, and $N_{f}$ pulses are used by the UWB-IR system to transmit such a codeword with bit-to-codeword mapping as $0 \rightarrow \mathbf{b}^{(1)}$ and $0 \rightarrow \mathbf{b}^{(2)}$. In particular, the transmitted signal in a point-to-point UWB-IR link with BC modulation is expressed as

$$
s(t)=\sum_{i=-\infty}^{\infty} \sum_{j=0}^{N_{f}-1} b_{i, j} \omega\left(t-i T_{s}-j T_{f}\right)
$$

where $\omega(t)$ is the energy-normalized monocycle pulse of duration $T_{\omega}, T_{f}$ and $T_{s}=N_{f} T_{f}$ are the frame and symbol durations, respectively, and $b_{i, j} \in\{ \pm 1\}$, modulating the pulse polarity, is the $j$ th element of the $i$ th transmitted codeword $\mathbf{b}_{i}=\left[b_{i, 0}, b_{i, 1}, \ldots, b_{i, N_{f}-1}\right]$, which is chosen from the codebook $\mathbf{B}_{2 \times N_{f}}$. Typically, different row vectors in $\mathbf{B}$ are orthogonal to guarantee good detection performance, and orthogonal $\mathrm{BC}$ implies that the codeword length is an even number; thus, $N_{f}$ is assumed to be even in this paper. The UWB multipath channel is described by a tap-delay line model as

$$
h(t)=\sum_{l=0}^{L-1} \alpha_{l} \delta\left(t-\tau_{l}\right)
$$

where the channel gain coefficients $\left\{\alpha_{l}\right\}$ and path delay coefficients $\left\{\tau_{l}\right\}$ of the MPCs are assumed to follow IEEE 802.15.3a [23]. $L$ is the number of the MPC, and the total path gains are normalized. To separate the multipath dispersion effects from the propagation delay $\tau_{0}$, all relative path delays can uniquely be cast into $\tau_{l, 0}:=\tau_{l}-\tau_{0}$, and $\left\{\tau_{l, 0}\right\}$ satisfies $\tau_{0,0}=$ $0, \tau_{l, 0}<\tau_{l+1,0}$. The channel is unknown to the receiver and is assumed to be quasistatic, which means that the channel remains invariant over one transmission burst but may change across bursts. Because this paper focuses on the optimized BC design, we assume perfect synchronization at the receiver side for simplicity, and the received signal is given as

$$
r(t)=\sum_{k=-\infty}^{\infty} b_{k} g\left(t-k T_{f}\right)+n(t)
$$

where $g(t)=\omega(t) * h(t)=\sum_{l=0}^{L-1} \alpha_{l} \omega\left(t-\tau_{l, 0}\right)$ is the overall channel impulse response with maximum waveform delay spread $T_{g}=\tau_{L-1,0}+T_{\omega}$, and $n(t)$ is a zero-mean additive Gaussian noise with two-sided power spectral density $N_{0} / 2$ over the system bandwidth $W$. Note that, for analytical convenience, we introduce the single time index $k=$ $i N_{f}+j$ in (3) to replace the double index tuple $(i, j)$ in (1) and the $i$ th transmitted codeword is rewritten as $\mathbf{b}_{i}=$ $\left[b_{i N_{f}}, b_{i N_{f}+1}, \ldots, b_{i N_{f}+N_{f}-1}\right]$. Before the detailed discussion on IFI/ISI, we briefly review the noncoherent CMSA receiver [11]. To begin with, some notations are introduced for ease of exposure. Let us define the $k$ th frame-long waveform of the received signal $r(t)$ in (3) as $r_{k}(t)=r\left(t+k T_{f}\right) G_{t \in\left[0, T_{f}\right)}$, where the gate function is defined as $G_{t \in\left[t_{1}, t_{2}\right)}=1$ and $G_{t \notin\left[t_{1}, t_{2}\right)}=0$. Based on the frame-long waveforms, the $i$ th 
symbol-long waveform $r\left(t+i T_{s}\right) G_{t \in\left[0, T_{s}\right)}$ is equivalently expressed by a waveform vector as

$$
\mathbf{r}_{i}(t):=\left[r_{i N_{f}}(t), r_{i N_{f}+1}(t), \ldots, r_{i N_{f}+N_{f}-1}(t)\right]^{T}
$$

where the components are the sequentially stacked frame-long waveforms of the $i$ th received symbol. With aforementioned notations, the decision strategy of BC-CMSA is expressed as

$$
\hat{\mathbf{b}}_{i}=\arg \max _{m \in\{1,2\}} \int_{0}^{T_{i}}\left[\mathbf{b}^{(m)} \mathbf{r}_{i}(t)\right]^{2} d t
$$

where $T_{I}$ is the integration interval. Based on (4), we can see that it takes the following two steps to get the decision variable: 1) the CMSA predetection operation or the linear filtering with the trial codeword $\mathbf{b}^{(m)} \mathbf{r}_{i}(t)$ and 2) the $\mathrm{ED} \int_{0}^{T_{I}}(\cdot)^{2} d t$. In addition, note that the CMSA predetection performed in (4) may require a digital receiver for practical implementation, which may be very complicated for conventional technology. However, in this paper, our main goal is to study the IFI/ISIrobust BC-CMSA scheme from the theoretical perspective, whereas the practical implementation of the proposed scheme is an interesting topic for future study.

In particular, the linear predetection operation $\mathbf{b}^{(m)} \mathbf{r}_{i}(t)$ is essential for the CMSA receiver. Due to the orthogonality among different codewords, the energy of the received signal can exclusively be aggregated by the right trial codeword along with the CMSA predetection in the absence of IFI/ISI, which leads to good detection performance. However, IFI/ISI will inevitably pollute the received signal, and the linear predetection operation will not exclusively aggregate signal energy for the right trial codeword, which results in a degraded detection performance. In the next section, we will investigate the mechanism of the BC-CMSA system in the presence of IFI/ISI to reveal the factor that influences performance.

\section{INTERFRAME INTERFERENCE/INTERSYMBOL INTERFERENCE ANALYSIS FOR BLOCK-CODE-CODEWORD MATCHING AND Signal AggRegation With the LAYERED INTERFERENCE MODEL}

In this section, the performance of the BC-CMSA system in the presence of IFI/ISI is analyzed. Note that IFI/ISI arises whenever the frame/symbol duration is shorter than the maximum waveform delay spread of the channel $T_{g}$. To explicitly show the interference involved in the received signal, we first rewrite (3) as

$$
r(t)=\sum_{k=-\infty}^{\infty} b_{k} \sum_{p=0}^{P} g_{p}\left(t-(k+p) T_{f}\right)+n(t)
$$

where $g(t)$ in (3) is partitioned into several frame-long and nonoverlapped segments as $g(t)=\sum_{p=0}^{P} g_{p}\left(t-p T_{f}\right)$, with $\left.g_{p}(t):=g\left(t+p T_{f}\right) G_{t \in\left[0, T_{f}\right.}\right)$, and $P=\left\lceil T_{g} / T_{f}\right\rceil-1$ is the number of following frames covered by the leaked overall channel response of the current frame. Fig. 1(a) illustrates the partition process with one realization of the CM2 channel [23]. Before proceeding, we would like to introduce the moderate IFI/ISI assumption that is used to ease the theoretical analysis.

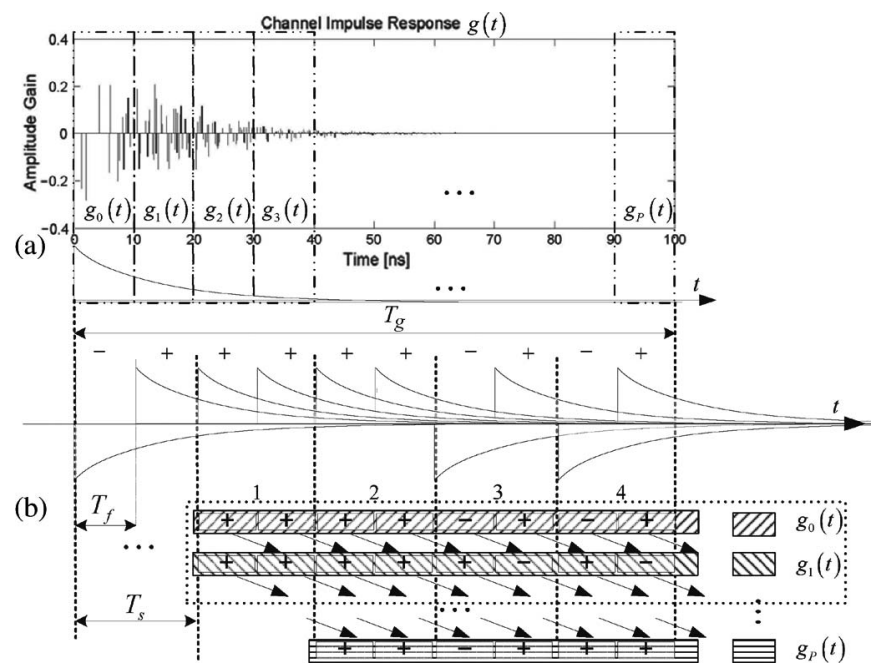

Fig. 1. (a) Overall channel response partition for one CM2 channel realization. (b) LI model with four AIPs.

We assume that the system is designed with a suitable frame duration $T_{f}$ and frame number $N_{f}$ and that the power of the overall channel response is mainly aggregated within $T_{s}$; therefore, ISI is considered between adjacent symbols, i.e., $g_{p}(t) \approx 0$ when $p>N_{f}-1$. In addition, if $T_{f}$ approaches the root mean square (RMS) delay $\tau_{R M S}$ of the considered channel, there exists strong interference only between adjacent frames. Based on these moderate IFI/ISI assumptions, we can develop the interference model with clarity. System performance in the presence of strong IFI/ISI will be shown in the simulation part.

\section{A. LI Model}

According to (4), the CMSA receiver makes decisions based on the waveform vector $\mathbf{r}_{i}(t)$ that corresponds to the $i$ th symbol-long received waveform. After some mathematical manipulations on (5), we can explicitly show the interference structure of $\mathbf{r}_{i}(t)$ as

$$
\mathbf{r}_{i}(t)=\sum_{p=0}^{N_{f}-1} \tilde{\mathbf{b}}_{i, p}^{T} g_{p}(t)+\mathbf{n}_{i}(t)
$$

where $\quad \tilde{\mathbf{b}}_{i, p}:=\left[b_{i N_{f}-p}, b_{i N_{f}+1-p}, \ldots, b_{i N_{f}+N_{f}-1-p}\right]$ is the p-frame-shifted version of the currently transmitted codeword $\mathbf{b}_{i}$, including the last $p$ elements of the preceding codeword $\mathbf{b}_{i-1}, \mathbf{n}_{i}(t):=\left[n_{i N_{f}}(t), n_{i N_{f}+1}(t), \ldots, n_{i N_{f}+N_{f}-1}(t)\right]^{T}$, and $g_{p}(t)=0$ when $p>N_{f}-1$ according to the adjacent ISI assumption. Notably, (6) describes a LI model. Corresponding to the channel response partition for $g(t)$, the IFI/ISI-polluted signal is decomposed into several superimposed layers by the LI model. The item behind the summation sign in (6) is considered to be the layer that consists of a layer indicator $g_{p}(t)$ and a codeword; for example, $\left[b_{i N_{f}}, b_{i N_{f}+1}, \ldots, b_{i N_{f}+N_{f}-1}\right]^{T} g_{0}(t)$ is the $p=0$ layer. The layer index $p$ can be treated as an energy indicator, and with a suitable $T_{f}$ setting, the layer with the smaller index statistically aggregates more signal energy than the layer with the larger index, which complies with the decay property of the average power delay profile for the UWB 
channel [12]. The $p=0$ layer is the IFI/ISI-free layer for the $i$ th symbol, in which the currently transmitted codeword $\tilde{\mathbf{b}}_{i, 0}=\mathbf{b}_{i}$ lies. Meanwhile, the codewords in the $p>0$ layers serve as interference to $\mathbf{b}_{i}$. Fig. 1(b) illustrates the LI model for an example. The $\mathrm{BC}$ matrix consists of two orthogonal codewords $[1,1]$ and $[-1,1]$. Each codeword can carry 1 bit of information, and IFI/ISI arises whenever $T_{s}<T_{g} . g(t)$ is depicted by an exponential envelop for clarity. The mechanism of interference is rather simple in the LI model. Treating the $p=0$ layer as the top layer, the codeword always shifts to a lower layer as time goes, with a step of $T_{f}$. The LI model gives a general description of IFI/ISI confronted by the CMSA receiver, which highlights the codeword structure. In the subsequent sections, we will continue the analysis based on the LI model.

\section{B. IFI/ISI Analysis With Channel-Averaged Approximation}

Recalling that the codeword index is $m \in\{1,2\}$ for $\mathbf{B}_{2 \times N_{f}}$, we further define the AIP as the codeword index pair of the adjacently transmitted codewords, i.e., $d=\left(m_{i-1}, m_{i}\right) \in$ $\{1,2\} \times\{1,2\} \forall i \in \mathbb{Z}$, where $m_{i}$ is the codeword index of the $i$ th transmitted codeword in the codebook, e.g., $\mathbf{b}_{i}=\mathbf{b}^{\left(m_{i}\right)}$. Based on the definition, the $p$-frame-shifted codeword $\tilde{\mathbf{b}}_{i, p}$ consists of elements from the adjacently transmitted codewords $\mathbf{b}_{i-1}$ and $\mathbf{b}_{i}$; therefore, $\tilde{\mathbf{b}}_{i, p}$ is a function of the AIP $d$. For brevity, $d$ is usually omitted in $\tilde{\mathbf{b}}_{i, p}(d)$, unless it is specially explained. Using the LI model in (6), the CMSA predetection signal is given by [cf., (4)]

$$
\mathbf{b}^{(m)} \mathbf{r}_{i}(t)=\mathbf{c}_{i}^{(m)} \mathbf{g}(t)+n_{i}^{(m)}(t), \quad m=1,2
$$

where $\mathbf{g}(t):=\left[g_{0}(t), g_{1}(t), \ldots, g_{N_{f}-1}(t)\right]^{T}$ contains the first $N_{f}$ segments of the partitioned $g(t), \mathbf{c}_{i}^{(m)}:=\left[c_{i, 1}^{(m)}, \ldots\right.$, $\left.c_{i, N_{f}-1}^{(m)}\right]$ contains all the correlation results between the trial codeword $\mathbf{b}^{(m)}$ and the real codewords within different layers in the LI model, i.e., $c_{i, p}^{(m)}:=\mathbf{b}^{(m)} \tilde{\mathbf{b}}_{i, p}^{T}$, and $n_{i}^{(m)}(t):=$ $\mathbf{b}^{(m)} \mathbf{n}_{i}(t)$ is the noise component in the predetection signal. The superscript $m$ in $\mathbf{c}_{i}^{(m)}$ and $n_{i}^{(m)}(t)$ indicates the codeword index of the trial codeword $\mathbf{b}^{(m)}$. Note that $\mathbf{c}_{i}^{(m)}$ is also a function of $d$, because $\tilde{\mathbf{b}}_{i, p}$ is involved. Based on (7), it is observed that the predetection signal $\mathbf{b}^{(m)} \mathbf{r}_{i}(t)$ is the weighted summation of the partial channel response in $\mathbf{g}(t)$, and the weighting coefficients are the elements of $\mathbf{c}_{i}^{(m)}$, which is determined by the codewords' correlation results. From the viewpoint of the LI model, these correlations will determine the power that was aggregated in different layers for the predetection signal.

For analytical convenience, we first ignore the noise component $n_{i}^{(m)}(t)$ in (7) and write the noise-free decision variable after ED as

$$
\begin{aligned}
\Lambda^{\prime}\left[\mathbf{r}_{i}(t) \mid \mathbf{b}^{(m)}\right] & =\int_{0}^{T_{f}}\left[\mathbf{c}_{i}^{(m)} \mathbf{g}(t)\right]^{2} d t \\
& =\mathbf{c}_{i}^{(m)} \mathbf{R}\left(\mathbf{c}_{i}^{(m)} t\right)^{T}, \quad m=1,2
\end{aligned}
$$

where the integration interval is set as $T_{I}=T_{f},[\mathbf{R}]_{p+1, p^{\prime}+1}=$ $\int_{0}^{T_{f}} g_{p}(t) g_{p^{\prime}}(t) d t$ is the element of $\mathbf{R}$ with $p$ and $p^{\prime} \in\left[0, N_{f}-\right.$ 1 , which is determined by instant channel realization. Because the final objective is to find the statistically optimal BC, we introduce the channel-averaged version of $\mathbf{R}$ as $\overline{\mathbf{R}}:=\mathrm{E}_{h}\{\mathbf{R}\}$, where $\mathrm{E}_{h}\{\cdot\}$ gets the expectation over all possible channel realizations. This way, the random properties of $\mathbf{R}$ is bypassed to simplify the analysis. Moreover, the expectation of the channel correlation functions is given by [24]

$$
\mathrm{E}_{h}\left\{\int_{\Omega} g(t) g(t-\Delta) d t\right\} \approx \phi_{\omega}(\Delta) \int_{\Omega} \psi_{h}(t) d t
$$

where $\phi_{\omega}(\Delta)=\int_{-\infty}^{\infty} \omega(t) \omega(t+\Delta) d t$ is the autocorrelation function of $\omega(t), \psi_{h}(t)$ is the average power delay profile, and $\Omega$ is the integration interval. By using (9), the entry of $\overline{\mathbf{R}}$ is given as

$$
\begin{aligned}
{[\overline{\mathbf{R}}]_{p+1, p^{\prime}+1} } & =\mathrm{E}_{h}\left\{\int_{0}^{T_{f}} g_{p}(t) g_{p^{\prime}}(t) d t\right\} \\
& \approx \phi_{\omega}\left(\left(p-p^{\prime}\right) T_{f}\right) \int_{p T_{f}}^{(p+1) T_{f}} \psi_{h}(t) d t .
\end{aligned}
$$

Because the support of $\omega(t)$ is only $\left[0, T_{\omega}\right), \phi_{\omega}\left(\left(p^{\prime}-p\right) T_{f}\right)=0$ whenever $p \neq p^{\prime}$, subsequently, $[\overline{\mathbf{R}}]_{p+1, p^{\prime}+1} \approx 0$ if $p \neq p^{\prime}$. Furthermore, when $p=p^{\prime}, \phi_{\omega}(0)=1$ for energy normalization; thus, we define $\bar{\varepsilon}_{p}:=[\overline{\mathbf{R}}]_{p+1, p+1}=\int_{p T_{f}}^{(p+1) T_{f}} \psi_{h}(t) d t$ as the averaged power of $g_{p}(t)$. Based on the aforementioned analysis, $\overline{\mathbf{R}}$ can be approximated as a diagonal matrix $\mathbf{D}=$ $\operatorname{diag}\left\{\bar{\varepsilon}_{0}, \bar{\varepsilon}_{1}, \ldots, \bar{\varepsilon}_{N_{f}-1}\right\}$. By replacing $\mathbf{R}$ in (8) with $\mathbf{D}$, the noise-free and channel-averaged decision variable is given as

$$
\begin{aligned}
\bar{\Lambda}^{\prime}\left[\mathbf{r}_{i}(t) \mid \mathbf{b}^{(m)}\right]: & =\mathrm{E}_{h}\left\{\Lambda^{\prime}\left[\mathbf{r}_{i}(t) \mid \mathbf{b}^{(m)}\right]\right\} \\
& \approx \sum_{p=0}^{N_{f}-1}\left(c_{i, p}^{(m)}\right)^{2} \bar{\varepsilon}_{p}, \quad m=1,2
\end{aligned}
$$

where $c_{i, p}^{(m)}$ is the $p$ th element of $\mathbf{c}_{i}^{(m)}$. We can see that the decision variable in (11) is the weighted summation of the averaged power contained in all layers and the weighting coefficients are squared elements of $\mathbf{c}_{i}^{(m)}$, which is also determined by the codeword's correlation properties. Therefore, the BC can be chosen so that the system performance can be optimized in the presence of IFI/ISI.

\section{SNR Analysis With IFI/ISI}

Distinct from the traditional signal-to-interference-plusnoise ratio (SINR) analysis, which treats the IFI/ISI as a certain kind of noise, we derive the signal-to-noise ratio (SNR) with IFI/ISI for the currently transmitted symbol conditioned on the AIP. Because the purpose of the analysis is not to develop the accurate closed-form expression of BER but to facilitate 
code optimization, all the derivations are based on the channelaveraged decision variable [cf., (11)]. In addition, the moderate IFI/ISI condition is used. With these assumptions, the decision rule for binary BC-CMSA is given as

$$
\bar{\Lambda}\left[\mathbf{r}_{i}(t) \mid \mathbf{b}^{(1)}\right] \underset{\mathbf{b}^{(2)}}{\stackrel{\mathbf{b}^{(1)}}{\gtrless}} \bar{\Lambda}\left[\mathbf{r}_{i}(t) \mid \mathbf{b}^{(2)}\right]
$$

and the decision variable that involves noise is expressed as

$$
\bar{\Lambda}\left[r_{i}(t) \mid \mathbf{b}^{(m)}\right] \approx \bar{S}_{i}^{(m)}+\bar{I}_{i, 1}^{(m)}+\bar{\xi}_{i, 1}^{(m)}+\bar{\xi}_{i, 2}^{(m)}, \quad m=1,2
$$

where each term in (13) is defined in Appendix A, and $\bar{S}_{i}^{(m)}$, $\bar{I}_{i, 1}^{(m)}, \bar{\xi}_{i, 1}^{(m)}$, and $\bar{\xi}_{i, 2}^{(m)}$ are all functions of the AIP. Based the results in (12) and (13), the probability of error that is conditioned on the AIP, $d^{\prime}=\left(m_{i-1}, m_{i}=m\right)$, is given by

$$
\operatorname{Pr}\left(\mathbf{b}^{\left(m_{i}=m\right)} \rightarrow \mathbf{b}^{\left(m^{\prime} \neq m\right)} \mid d^{\prime}\right)=\operatorname{Pr}\left(\bar{X}_{i}\left(d^{\prime}\right)<0\right)
$$

and the random variable $\bar{X}_{i}\left(d^{\prime}\right)$ is defined as

$$
\begin{aligned}
\bar{X}_{i}\left(d^{\prime}\right): & =\bar{\Lambda}\left[\mathbf{r}_{i}(t) \mid \mathbf{b}^{(m)}\right]-\bar{\Lambda}\left[\mathbf{r}_{i}(t) \mid \mathbf{b}^{\left(m^{\prime}\right)}\right] \\
& \approx \bar{X}_{i, S}\left(d^{\prime}\right)+\bar{X}_{i, \xi}\left(d^{\prime}\right)
\end{aligned}
$$

where the signal component $\bar{X}_{i, S}\left(d^{\prime}\right)$ is expressed as

$$
\bar{X}_{i, S}\left(d^{\prime}\right)=\bar{S}_{i}^{(m)}\left(d^{\prime}\right)-\bar{S}_{i}^{\left(m^{\prime}\right)}\left(d^{\prime}\right)+\bar{I}_{i, 1}^{(m)}\left(d^{\prime}\right)-\bar{I}_{i, 1}^{\left(m^{\prime}\right)}\left(d^{\prime}\right) .
$$

and the noise component $\bar{X}_{i, \xi}\left(d^{\prime}\right)$ is expressed as

$$
\bar{X}_{i, \xi}\left(d^{\prime}\right)=\bar{\xi}_{i, 1}^{(m)}\left(d^{\prime}\right)-\bar{\xi}_{i, 1}^{\left(m^{\prime}\right)}\left(d^{\prime}\right)+\bar{\xi}_{i, 2}^{(m)}\left(d^{\prime}\right)-\bar{\xi}_{i, 2}^{\left(m^{\prime}\right)}\left(d^{\prime}\right)
$$

The mean and variance of $\bar{X}_{i}\left(d^{\prime}\right)$ is derived in Appendix B with similar procedures as in [6] and [22], and only the energy dominating layers $(p=0,1)$ is considered to highlight the major performance-influencing factors. With these statistics, the average receive SNR of the $i$ th symbol with IFI and ISI is defined in (16), shown at the bottom of the page, where $\lambda=\bar{\varepsilon}_{0} / \bar{\varepsilon}_{g}$ is the ratio between the averaged energy of $g_{0}(t)$ and $g(t)$, and $\mu=1-\lambda$. The subscript $n$ of $\mathrm{E}_{n}\{\cdot\}$ and $\operatorname{Var}_{n}\{\cdot\}$ indicates that the statistical analysis is for the noise component in $\bar{X}_{i}\left(d^{\prime}\right)$. In addition, $c_{i, 1}^{(m)}\left(d^{\prime}\right)=\mathbf{b}^{(m)}\left(\tilde{\mathbf{b}}_{i, 1}\left(d^{\prime}\right)\right)^{T}$ is the correlation between the trial codeword $\mathbf{b}^{(m)}$ and the major interfering codeword $\tilde{\mathbf{b}}_{i, 1}$ in the $p=1$ layer.

Note that $\mu=0$ and $\lambda=1$ in the absence of IFI/ISI. By replacing $\bar{\varepsilon}_{g}$ with the received energy per pulse $E_{p}=$
$\int_{0}^{T_{I}^{\prime}} g^{2}(t) d t$, where $T_{I}^{\prime}$ is the integral interval, (16) is rewritten as

$$
\gamma_{i}^{\prime}=\frac{\left\{N_{f}^{2} E_{p}\right\}^{2}}{2 N_{0} N_{f}^{3} E_{p}+2 N_{0}^{2} N_{f}^{2} T_{i}^{\prime} W}=\left(\frac{2 N_{0}}{E_{b}^{\prime}}+\frac{2 N_{0}^{2} T_{i}^{\prime} W}{E_{b}^{\prime 2}}\right)^{-1}
$$

where $E_{b}^{\prime}:=N_{f} E_{p}$ is the received energy per bit. The result in (17) is consistent with [22], but (16) includes the effects of IFI and ISI and, thus, is more general.

\section{CCG in the Presence of IFI/ISI}

In this section, we will reveal the intrinsic connection between the adopted BC and system performance by extracting the CCG from $\gamma_{i}\left(d^{\prime}\right)$. For convenient notation, we define SNR $:=E_{b} / N_{0}$ as the transmit SNR, where $E_{b}:=N_{f} \bar{\varepsilon}_{g}$. As proved in Appendix C, when SNR is high, the receive SNR $\gamma_{i}\left(d^{\prime}\right)$ can approximately be expressed as

$$
\gamma_{i}\left(d^{\prime}\right) \approx \frac{\mathbf{S N R}}{2} \beta_{i}\left(d^{\prime}\right)
$$

where

$$
\beta_{i}\left(d^{\prime}\right)=\frac{\left\{\lambda+\frac{\mu}{N_{f}^{2}}\left[\left(c_{i, 1}^{(m)}\left(d^{\prime}\right)\right)^{2}-\left(c_{i, 1}^{\left(m^{\prime}\right)}\left(d^{\prime}\right)\right)^{2}\right]\right\}^{2}}{\lambda+\frac{\mu}{N_{f}^{2}}\left[\left(c_{i, 1}^{(m)}\left(d^{\prime}\right)\right)^{2}+\left(c_{i, 1}^{\left(m^{\prime}\right)}\left(d^{\prime}\right)\right)^{2}\right]}
$$

is defined as the CCG that was conditioned on $d^{\prime}$. It can be observed that $\gamma_{i}\left(d^{\prime}\right)$ linearly increases with $\beta_{i}\left(d^{\prime}\right)$, which is a function of the codeword correlation $c_{i, 1}^{(m)}\left(d^{\prime}\right)$ and $c_{i, 1}^{\left(m^{\prime}\right)}\left(d^{\prime}\right)$; therefore, $\gamma_{i}\left(d^{\prime}\right)$ or the system detection performance is BC dependent in the presence of IFI/ISI. Moreover, the AIP $d^{\prime}$ is a random variable with probability $\operatorname{Pr}\left(d^{\prime}\right)=1 / 4, \forall d^{\prime} \in\{1,2\} \times$ $\{1,2\}$, according to the assumption of the equiprobable information source. Therefore, a BC-dependent average CCG is introduced by averaging $\beta_{i}\left(d^{\prime}\right)$ over all the possible $d^{\prime}$ s as

$$
\beta_{B C}:=\mathrm{E}_{d^{\prime}}\left\{\beta_{i}\left(d^{\prime}\right)\right\}
$$

which serves as an average performance metric for the BCCMSA system using different BCs; here, the symbol index $i$ is omitted for the ergodicity of $d^{\prime}$.

Based on the aforementioned analysis, we conclude that the $\mathrm{BC}$ can be optimized with respect to the average $\mathrm{CCG} \beta_{B C}$ to improve the detection performance in the presence of IFI/ISI. To this end, the upper bound of $\beta_{B C}$ and the conditions to reach the upper bound are of great interest. In fact, if there is

$$
\gamma_{i}\left(d^{\prime}\right):=\frac{\mathrm{E}_{n}^{2}\left\{\bar{X}_{i, S}\left(d^{\prime}\right)\right\}}{\operatorname{Var}_{n}\left\{\bar{X}_{i, \xi}\left(d^{\prime}\right)\right\}} \approx \frac{\left\{\lambda N_{f}^{2}+\mu\left[\left(c_{i, 1}^{(m)}\left(d^{\prime}\right)\right)^{2}-\left(c_{i, 1}^{\left(m^{\prime}\right)}\left(d^{\prime}\right)\right)^{2}\right]\right\}^{2} \bar{\varepsilon}_{g}^{2}}{2 N_{0} N_{f} \bar{\varepsilon}_{g}\left\{\lambda N_{f}^{2}+\mu\left[\left(c_{i, 1}^{(m)}\left(d^{\prime}\right)\right)^{2}+\left(c_{i, 1}^{\left(m^{\prime}\right)}\left(d^{\prime}\right)\right)^{2}\right]\right\}+2 N_{0}^{2} N_{f}^{2} T_{i} W}
$$


no IFI/ISI, the $p=0$ layer in the LI model can collect all the energy of $g(t)$, i.e., $\lambda=1, \mu=0$, and then, $\beta_{i}\left(d^{\prime}\right)=1, \forall d^{\prime}$, and $\beta_{B C}=1$. When IFI/ISI exists, the signal energy leaks into subsequent frames/symbols, and then, we can straightforwardly conclude that the conditional CCG is upper bounded by 1 , i.e., $\beta_{B C} \leq 1$. For analytical purposes, we first reformulate the upper bound of $\beta_{i}\left(d^{\prime}\right)$ as follows:

$$
\beta_{i}\left(d^{\prime}\right) \leq \lambda+\frac{\mu\left(c_{i, 1}^{(m)}\left(d^{\prime}\right)\right)^{2}}{N_{f}^{2}} \leq 1
$$

where the first inequality is obvious [see (19)], and only when $c_{i, 1}^{\left(m^{\prime}\right)}\left(d^{\prime}\right)=0$ does the equation hold. The second inequality holds with the condition $\left(c_{i, 1}^{(m)}\left(d^{\prime}\right)\right)^{2} \leq N_{f}^{2}$, and only when $c_{i, 1}^{(m)}\left(d^{\prime}\right)= \pm N_{f}$ does the equation hold. In summary, if the BC meets the conditions

$$
c_{i, 1}^{\left(m^{\prime}\right)}\left(d^{\prime}\right)=0 \text { and } c_{i, 1}^{(m)}\left(d^{\prime}\right)= \pm N_{f} \quad \forall d^{\prime}
$$

the conditional CCG reaches its upper bound as $\beta_{i}\left(d^{\prime}\right)=$ $1, \forall d^{\prime}$, and the average CCG is maximized as $\beta_{B C}=1$, even in the presence of IFI/ISI. Here, $c_{i, 1}^{\left(m^{\prime}\right)}\left(d^{\prime}\right)=\mathbf{b}^{\left(m^{\prime}\right)}\left(\tilde{\mathbf{b}}_{i, 1}\left(d^{\prime}\right)\right)^{T}$ is the correlation between the wrong trial codeword $\mathbf{b}^{\left(m^{\prime}\right)}$ and the major interfering codeword $\tilde{\mathbf{b}}_{i, 1}\left(d^{\prime}\right)$ in the $p=1$ layer, and $c_{i, 1}^{(m)}\left(d^{\prime}\right)=\mathbf{b}^{(m)}\left(\tilde{\mathbf{b}}_{i, 1}\left(d^{\prime}\right)\right)^{T}$ is the correlation between the right trial codeword $\mathbf{b}^{\left(m^{\prime}\right)}$ and $\tilde{\mathbf{b}}_{i, 1}\left(d^{\prime}\right)$. In the next section, attempts are made to construct the optimal BC that fulfills the conditions (22) with maximum $\beta_{B C}=1$.

\section{Optimized Block Code With InTERframe INTERFERENCE AND INTERSYMBOL INTERFERENCE PREMITIGATION}

Originally, BC-CMSA is developed under the condition of no IFI/ISI. Signal energy is aggregated in the $p=0$ layer of the LI model, and the orthogonality of $\mathrm{BC}$ alone can ensure good detection performance. However, IFI/ISI occurs with a boosted data rate. The interference results in $\tilde{\mathbf{b}}_{i, p}$ in the $p>0$ layer, particularly $\tilde{\mathbf{b}}_{i, 1}$ in the $p=1$ layer, is the main interference component, which should also be considered to enhance the detection performance. In this section, the desired IFI/ISIrobust code properties for the optimal $\mathrm{BC}$ are first generalized, which enable the maximum average CCG $\beta_{B C}=1$. Then, the optimal BC is constructed under the constraints of the desired code properties, followed by an implementation example to bring forward the premitigation of IFI and ISI.

\section{A. Construction of the Optimal BC}

With the aim of generalizing (22) to more insightful code properties, we first introduce some notations for ease of analysis. The codewords in the top two layers of the LI model vary according to the AIP, and all the realizations of the two codewords are classified into two sets as $\mathbf{A}_{1}=\left\{\mathbf{b}^{\left(m_{i}=1\right)}\right.$, $\left.\tilde{\mathbf{b}}_{i, 1}\left(m_{i-1}, m_{i}=1\right)\right\}$ and $\mathbf{A}_{2}=\left\{\mathbf{b}^{\left(m_{i}=2\right)}, \tilde{\mathbf{b}}_{i, 1}\left(m_{i-1}, m_{i}=\right.\right.$ $2)\}$. The set $\mathbf{A}_{m}$ "snapshots" the interference patterns of the currently transmitted codeword. With these notations, the desired IFI/ISI-robust code properties for the optimal BC are listed as follows.

1) Shifted orthogonality: $\mathbf{b}^{\left(m^{\prime}\right)} \mathbf{a}^{T}=0, \forall \mathbf{a} \in \mathbf{A}_{m}, m, m^{\prime} \in$ $\{1,2\}, m \neq m^{\prime}$.

2) Shifted repetition: $\mathbf{b}^{(m)} \mathbf{a}^{T}= \pm N_{f}, \forall \mathbf{a} \in \mathbf{A}_{m}, \quad m \in$ $\{1,2\}$.

To exemplify the meaning of shifted orthogonality and shifted -repetition, a lemma is first given as follows.

Lemma 1: Shifted orthogonality and shifted repetition are sufficient for the $\mathrm{BC}$ to achieve the maximum average CCG $\beta_{B C}=1$.

Proof: Simply verify (22) with the two code properties. If the transmitted symbol is $\mathbf{b}^{\left(m_{i}=m\right)}$, with shifted repetition, $c_{i, 1}^{(m)}\left(d^{\prime}\right)= \pm N_{f}$ for $m_{i}=m$, and with shifted orthogonality, $c_{i, 1}^{\left(m^{\prime}\right)}\left(d^{\prime}\right)=0$ for $m_{i}=m \neq m^{\prime}$. Then, $\beta\left(d^{\prime}\right)=1$ for arbitrary $d^{\prime}$; therefore, $\beta_{B C}=1$.

Based on Lemma 1, we can see the essence of the two properties: shifted orthogonality ensures zero correlation between the wrong trial codeword and the moderate IFI/ISI-polluted receive codeword, whereas shifted repetition ensures full correlation between the right trial codeword and the moderate IFI/ISIpolluted receive codeword. To this end, the optimal $\mathrm{BC}$ with shifted orthogonality and shifted repetition will enable robust detection performance in the presences of IFI/ISI.

Remark 1: Usually, the ordinary orthogonal $\mathrm{BC}$ does not hold shifted orthogonality and shifted repetition. For explanation convenience, we take the $\mathrm{BC}$ based on the Walsh-Hardmard matrix $\mathbf{B}_{2 \times 2}=\left[\begin{array}{cc}1 & 1 \\ 1 & -1\end{array}\right]$ as an example. Assume that the currently transmitted codeword is $\mathbf{b}^{\left(m_{i}=2\right)}=$ $[1,-1]$ and the adjacent inference pattern is $d=(1,2)$. Then, $\tilde{\mathbf{b}}_{i, 1}(1,2)=[1,1]$, and $\mathbf{A}_{2}=\left\{\mathbf{b}^{\left(m_{i}=2\right)}, \tilde{\mathbf{b}}_{i, 1}(1,2)\right\}$ is constructed. By using the wrong trial codeword $\mathbf{b}^{(1)}=[1,1]$, we get $\mathbf{b}^{(1)}\left(\mathbf{b}^{\left(m_{i}=2\right)}\right)^{T}=0$, but $\mathbf{b}^{(1)}\left(\tilde{\mathbf{b}}_{i, 1}(1,2)\right)^{T}=2$. On the other hand, by using the right codeword $\mathbf{b}^{(2)}=[1,-1]$, we have $\mathbf{b}^{(2)}\left(\mathbf{b}^{\left(m_{i}=2\right)}\right)^{T}=2$, but $\mathbf{b}^{(2)}\left(\tilde{\mathbf{b}}_{i, 1}(1,2)\right)^{T}=0$. In this case, $\mathbf{b}^{(1)}\left(\tilde{\mathbf{b}}_{i, 1}(1,2)\right)^{T}=2 \neq 0$ for the lack of shifted orthogonality and $\mathbf{b}^{(2)}\left(\tilde{\mathbf{b}}_{i, 1}(1,2)\right)^{T}=0 \neq \pm 2$ for the lack of shifted repetition, which means that the interference component $\tilde{\mathbf{b}}_{i, 1}(1,2)$ makes no contribution to support the right trial codeword $\mathbf{b}^{(2)}$ but full contribution to the wrong trial codeword $\mathbf{b}^{(1)}$. Consequently, the detection performance is degraded.

Constrained by the two code properties, we then give the construction of the optimal $\mathrm{BC}$ in the following lemma.

Lemma 2: $\widehat{\mathbf{B}}_{2 \times N_{f}}=\left[\widehat{\mathbf{B}}_{2 \times 2}, \ldots, \widehat{\mathbf{B}}_{2 \times 2}\right]$, where $\widehat{\mathbf{B}}_{2 \times 2}=$ $\left[\begin{array}{cc}1 & 1 \\ -1 & 1\end{array}\right]$ is the only bipolar $\mathrm{BC}$ with shifted orthogonality and shifted repetition.

Proof: See Appendix D.

Based on Lemmas 1 and 2, we show the optimality of the proposed $\widehat{\mathbf{B}}_{2 \times N_{f}}$ in the following proposition.

Proposition: $\widehat{\mathbf{B}}_{2 \times N_{f}}$ is the optimal $\mathrm{BC}$ with maximum $\beta_{B C}=1$.

Proof: Combining Lemma 1 with Lemma $2, \widehat{\mathbf{B}}_{2 \times N_{f}}$ is the unique $\mathrm{BC}$ with maximum $\beta_{B C}=1$; therefore, the proposition holds. 
Remark 2: Although there are $2^{N_{f}}$ possible codewords with code length $N_{f}$ and $C_{2}^{2^{N_{f}}}$ realizations of possible BCs, based on our construction, only $\widehat{\mathbf{B}}_{2 \times N_{f}}$ enjoys shifted orthogonality and shifted repetition, which contains only two codewords and can support binary modulation only. High-order modulation requires a codebook with a larger size, but based on the uniqueness of $\widehat{\mathbf{B}}_{2 \times N_{f}}$, we conclude that any attempt to construct $\widehat{\mathbf{B}}_{M \times N_{f}}(M>2)$ for high-order modulation with the maximum average CCG $\beta_{B C}=1$ will fail.

Remark 3: BC-CMSA can achieve $M$-ary $(M>2)$ modulation. Although there is no optimal $\mathrm{BC}$ of larger size to support high-order modulation with the average CCG $\beta_{B C}=1$ in the presence of moderate IFI/ISI, we can still employ (20) as an effective metric to search for the $\mathbf{B}_{M \times N_{f}}$ with a higher average CCG to benefit transmission. In this scenario, the AIP is extended as $d \in\{1,2, \ldots, M\} \times\{1,2, \ldots, M\}$, and more computation is involved to calculate (20).

\section{B. Optimized BC With IFI and ISI Premitigation: Implementation Example}

Codeword shifting happens across layers in the LI model. Within the detection window, the codeword in the $p=1$ layer is always a one-frame shifted version of the currently transmitted codeword. For shifted orthogonality, the $p=1$ layer will not aggregate energy for the undesired symbol; thus, ISI can greatly be mitigated. On the other hand, for shifted repetition, the $p=1$ layer will always help aggregate energy for the desired symbol; thus, IFI/ISI is partially utilized. This way, the premitigation on IFI/ISI is achieved before ED. Fig. 2 gives an implementation example to explain the premitigation effect with $\mathbf{B}_{2 \times 2}$. One of the four AIPs in Fig. 1(b) is zoomed in, and only the energy-dominating layers, i.e., the $p=0,1$ layers, are depicted. In addition, the exponential envelop is replaced by a triangle for clarity. The currently transmitted codeword is $\mathbf{b}^{\left(m_{i}=2\right)}=[-1,1]$, and the adjacent inference pattern is $d=$ $(1,2)$. Then, $\tilde{\mathbf{b}}_{i, 1}(1,2)=[1,-1]$. When testing $\mathbf{b}^{(2)}=[-1,1]$, $\mathbf{b}^{(2)}\left(\tilde{\mathbf{b}}_{i, 0}(1,2)\right)^{T}=2$, and $\mathbf{b}^{(2)}\left(\tilde{\mathbf{b}}_{i, 1}(1,2)\right)^{T}=-2$, after ED, both layers are contributed to the desired symbol. Due to shifted repetition, the energy of the leaked signal can partially be used to enhance the detection performance, as pointed out by Effect 1 in Fig. 2. Meanwhile, both $\tilde{\mathbf{b}}_{i, 0}(1,2)$ and $\tilde{\mathbf{b}}_{i, 1}(1,2)$ are orthogonal with the wrong trial codeword $\mathbf{b}^{(1)}=[1,1]$; thus. IFI/ISI is greatly mitigated before the ED by shifted orthogonality, as pointed out by Effect 2 in Fig. 2. In summary, the optimized $\mathrm{BC}$ tailors the AIP to the CMSA predetection operation so that the energy of the main interfering component in the $p=1$ layer can be aggregated only for the desired codeword before the ED; thus, IFI/ISI premitigation is achieved.

\section{Simulation Results and Discussions}

In this section, simulations and comparisons are carried out to validate that the proposed code-optimized $\mathrm{BC}$ (CO-BC) $\widehat{\mathbf{B}}_{2 \times N_{f}}$ achieves good IFI and ISI premitigation with CMSA. The impulse shape that was used is $\omega(t)=A_{H}[1-$ $\left.4 \pi\left(t / \tau_{m}\right)^{2}\right] \exp \left[-2 \pi\left(t / \tau_{m}\right)^{2}\right]$, where $A_{H}$ is the energynormalized parameter, and $\tau_{m}=0.2877 \mathrm{~ns}$ so that the pulse

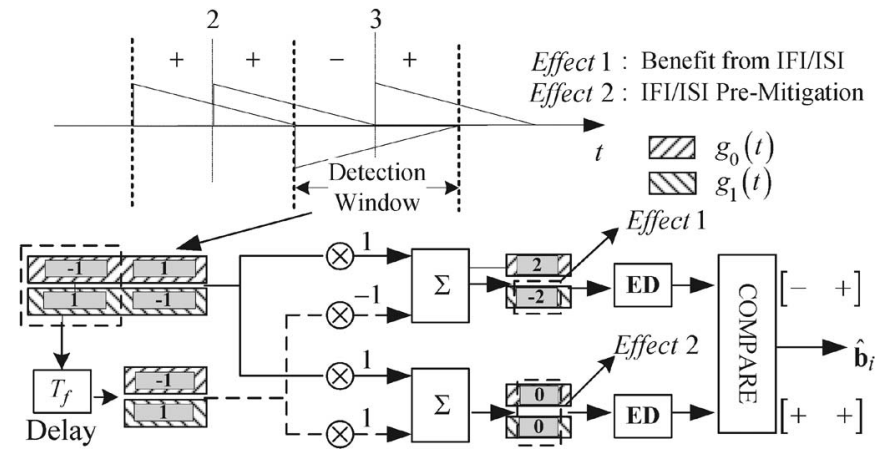

Fig. 2. IFI and ISI premitigation with the optimized BC.

width is limited within $1 \mathrm{~ns}$. In all cases, the channels are generated according to the IEEE 802.15.3a channel model recommendation CM2 with an RMS delay of $\tau_{R M S}=8.06 \mathrm{~ns}$ [12], and the channel impulse responses are truncated beyond $T_{g}=100 \mathrm{~ns}$ with energy normalization. We also set $T_{I}=T_{f}$, unless it is specially explained.

Test Case 1: This test case is devoted to demonstrating the effectiveness of the CO-BC for BC-CMSA in the presence of IFI/ISI. Signal structures for the binary modulation schemes are set as $N_{f}=2$ frames (with frame duration $T_{f}=10 \mathrm{~ns}$ ) and $N_{f}=4$ frames (with frame duration $T_{f}^{\prime}=5 \mathrm{~ns}$ ) per bit with the same symbol duration $T_{s}=20 \mathrm{~ns}$ or, equivalently, a data rate of $50 \mathrm{Mb} / \mathrm{s}$. A 4-ary modulation scheme is also considered for BC-CMSA with the same data rate as the binary cases, which exploits a larger BC $\mathbf{B}_{4 \times 4}$ and conveys 2 bits of information per codeword with symbol duration $T_{s}^{\prime}=4 T_{f}=40 \mathrm{~ns}$. The Walsh-Hardmard matrices $\mathbf{H}_{2}=\left[\begin{array}{cc}1 & 1 \\ 1 & -1\end{array}\right]$ and $\mathbf{H}_{4}=\mathbf{H}_{2} \otimes$ $\mathbf{H}_{2}$ are exploited for schemes based on Walsh-BC. In particular, we use the first and the second rows of $\mathbf{H}_{4}$, denoted as $\mathbf{H}_{4}(1,2)$, for binary BC-CMSA with code length $N_{f}=4$. In addition, the IFI/ISI-free condition is realized by truncating the channel at 9 ns with energy normalization, and the corresponding simulation results are shown as benchmarks for comparison. Fig. 3 shows that the performances of $\mathbf{H}_{2}, \mathbf{H}_{4}(1,2)$, and CO$\mathrm{BC}$ are nearly the same with binary modulation and the result with $\mathbf{H}_{4}$-BC is better with 4-ary modulation under the IFI/ISIfree condition. Assuming the same transmit energy per bit, $\mathbf{H}_{4}$-BC enables 4-ary modulation with increased minimum distance between codewords; therefore, better BER performance is achieved compared with the binary cases. In addition, the frame number or code length $N_{T}$ dose not influence the performance of the binary BC-CMSA if the same bit energy and data rate is assumed in the absence of IFI/ISI. However, it is found that there are distinct gaps between the IFI/ISI-existing and IFI/ISI-free cases for $\mathbf{H}_{2}-\mathrm{BC}$ and $\mathbf{H}_{4}$-BC. On the other hand, the proposed CO-BC with code length $N_{T}=2$ achieves comparable performance as its interference-free counterpart in the presence of IFI/ISI, losing only about $1 \mathrm{~dB}$ at BER = $10^{-3}$. The results indicate that the orthogonal BCs, such as $\mathbf{H}_{2}$ and $\mathbf{H}_{4}$-BC, cannot eliminate or even utilize the leaked signal energy, and thus, they are very sensitive to IFI/ISI. On the contrary, the proposed CO-BC with shifted orthogonality and shifted repetition can achieve IFI/ISI premitigation with 


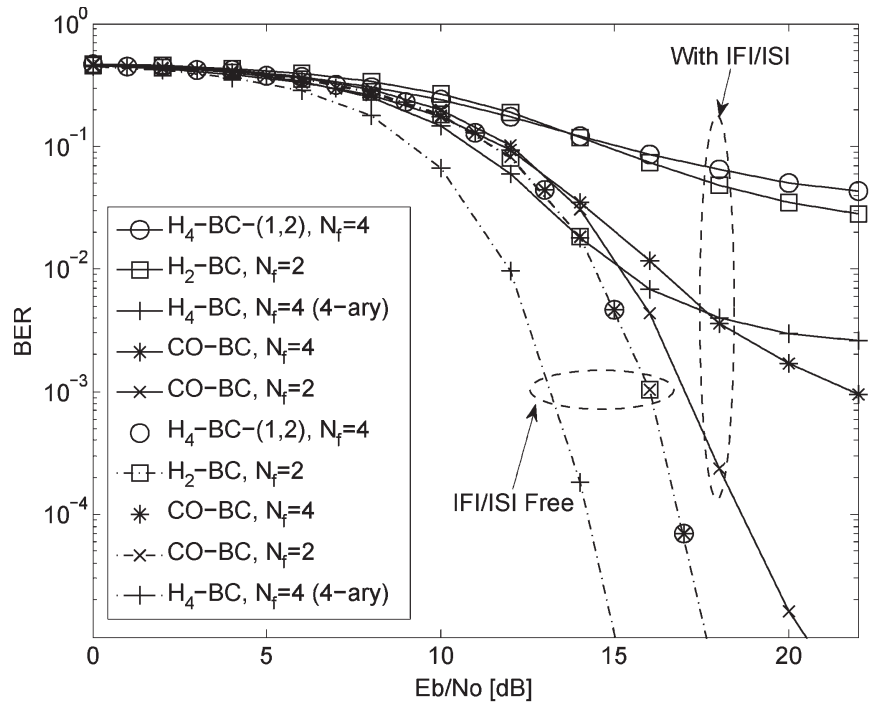

Fig. 3. BER performance comparison between the CO-BC and Walsh-Hardmard-based BC-CMSA, where $T_{f}=10 \mathrm{~ns}$ is set for all schemes, and the default modulation order is set to 2 , unless otherwise specified in the legend.

TABLE II

CCGS FOR DIFFERENT BCS

\begin{tabular}{cccccc}
\hline$B C / C G$ & $\beta(1,1)$ & $\beta(2,1)$ & $\beta(1,2)$ & $\beta(2,2)$ & $\beta_{B C}$ \\
\hline $\mathbf{H}_{4}-B C(1,2)$ & 1.000 & 0.592 & 0.592 & 1.000 & 0.796 \\
$\overline{\mathbf{H}}_{4}-B C(1,3)$ & 1.000 & 0.592 & 0.592 & 1.000 & 0.724 \\
$\mathbf{H}_{4}-B C(1,4)$ & 1.000 & 1.000 & 0.712 & 0.712 & 0.856 \\
$\overline{\mathbf{H}}_{4}-B C(2,3)$ & 1.000 & 1.000 & 0.712 & 0.712 & 0.856 \\
$\overline{\mathbf{H}}_{4}-B C(2,4)$ & 1.000 & 0.592 & 0.592 & 1.000 & 0.724 \\
$\mathbf{H}_{4}-B C(3,4)$ & 0.179 & 0.592 & 0.592 & 0.179 & 0.386 \\
\hline$C O-B C$ & 1.000 & 1.000 & 1.000 & 1.000 & 1.000 \\
\hline
\end{tabular}

CMSA and shows good robustness to IFI/ISI. It is also noted that the performance of CO-BC with code length $N_{T}=4$ is worse than with $N_{T}=2$ in the presence of IFI/ISI. This case is simply because more frames or a longer code length per symbol increases IFI/ISI, assuming the same data rate. In addition, the shortest code length $N_{T}=2$ is optimal for high-data-rate BC-CMSA.

Test Case 2: The average CCG $\beta_{B C}$ 's deterministic influence on the system performance in the presence of moderate IFI/ISI is illustrated, and the optimality of the proposed CO-BC is also verified in this test case. In accordance with moderate IFI/ISI assumption, $T_{f}=10 \mathrm{~ns}$ and $T_{s}=N_{f} T_{f}=40 \mathrm{~ns}$ are set for binary modulation, which achieves a data rate of $25 \mathrm{Mb} / \mathrm{s}$. Because $T_{s} \approx 5 \tau_{R M S}$, the adjacent ISI assumption can approximately hold. The Walsh-Hardmard $\mathbf{H}_{4}$ is considered a typical codebook for orthogonal $\mathrm{BC}$, and the combinations of every two rows of $\mathbf{H}_{4}$ generate multiple orthogonal BCs for binary modulation; for example, $\mathbf{H}_{4}(1,2)$ consists of the first and second rows of $\mathbf{H}_{4}$. To get correspondent $\beta_{B C}$, the ratio $\lambda=\bar{\varepsilon}_{0} / \bar{\varepsilon}_{g}$ is first given through simulation. Two thousand CM2 channel realizations are simulated and analyzed by the computer, and $\left[\bar{\varepsilon}_{0}, \bar{\varepsilon}_{1}\right] \approx[0.7118,0.2221]$ is obtained. Note that the channel energy is normalized and $\bar{\varepsilon}_{0}+\bar{\varepsilon}_{1} \approx \bar{\varepsilon}_{g}=1$; therefore, $\lambda \approx 0.7118$ is used. With these parameters, both the conditional CCG $\beta_{i}\left(d^{\prime}\right)$ and the average CCG $\beta_{B C}$ can be calculated from (19) and (20). The results are given in Table II, and it can

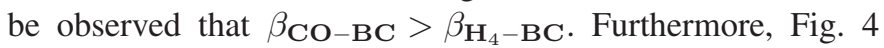

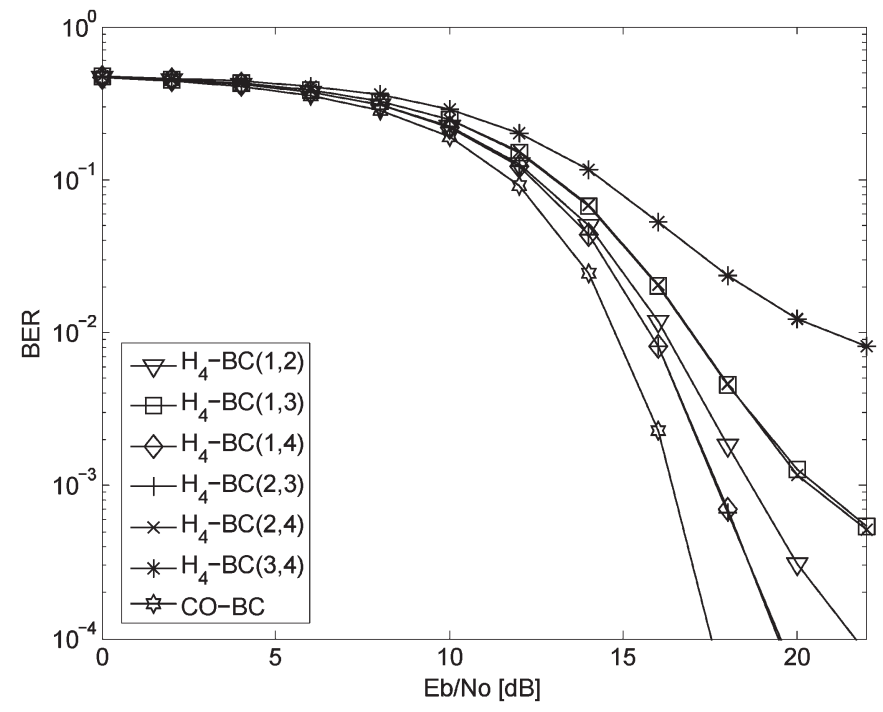

Fig. 4. Effects of the average CCG on the system performance of BC-CMSA in the presence of moderate IFI/ISI, where $N_{f}=4$ and $T_{f}=10 \mathrm{~ns}$ are set so that $\tau_{R M S}<T_{f}<2 \tau_{R M S}$ and $T_{s} \approx 5 \tau_{R M S}$.

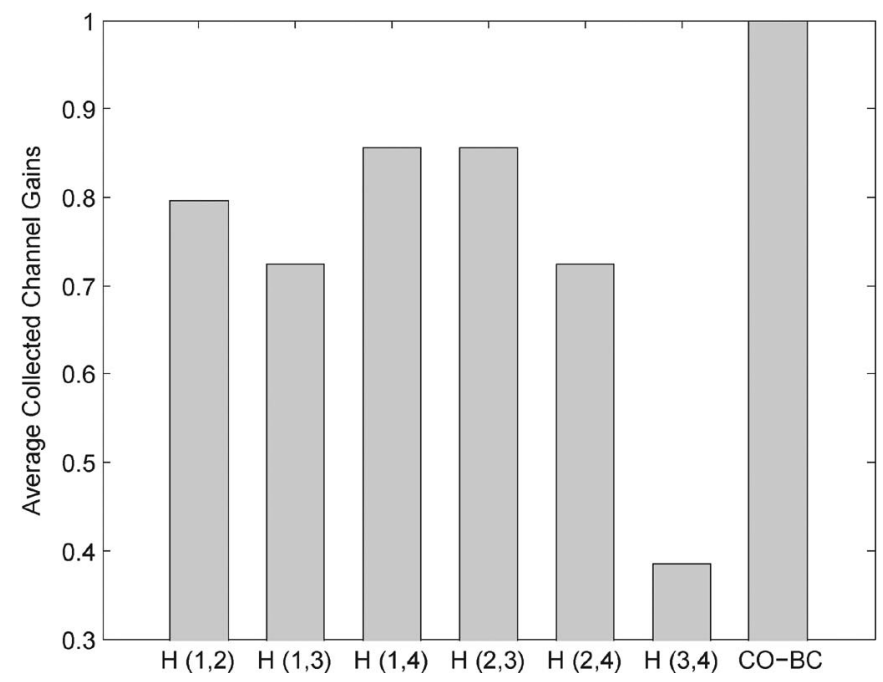

Fig. 5. Average CCGs for different BCs in the presence of moderate IFI/ISI, where $N_{f}=4$ and $T_{f}=10 \mathrm{~ns}$ are set for all schemes.

reveals the actual BER performance of the BC-CMSA system employing different BCs, and Fig. 5 shows the corresponding average CCGs for these BCs. Corresponding to the results in Table II, when $\beta_{\mathrm{BC}}$ is higher, the system shows better BER performance, and $\mathrm{CO}-\mathrm{BC}$ achieves the best performance with the maximum average $\mathrm{CCG} \beta_{\mathbf{C O}-\mathbf{B C}}=1$. The simulation results in Figs. 4 and 5 consolidate the average CCG analysis for different BCs. Finally, the moderate IFI/ISI constraint is relaxed, and $T_{f}=5 \mathrm{~ns}$ is set. The IFI become severe, and the effective ISI may disperse across more than two symbols. As shown in Fig. 6, the optimality of the proposed CO-BC still holds, whereas the performance gaps between $\mathrm{CO}-\mathrm{BC}$ and other $\mathbf{H}_{4}$ - $\mathrm{BCs}$ become even larger. These results demonstrate that, for ED-based BC-CMSA, the proposed CO-BC enjoys the best robustness under moderate IFI/ISI and the optimality holds, even in the presence of severe IFI and strong ISI. 


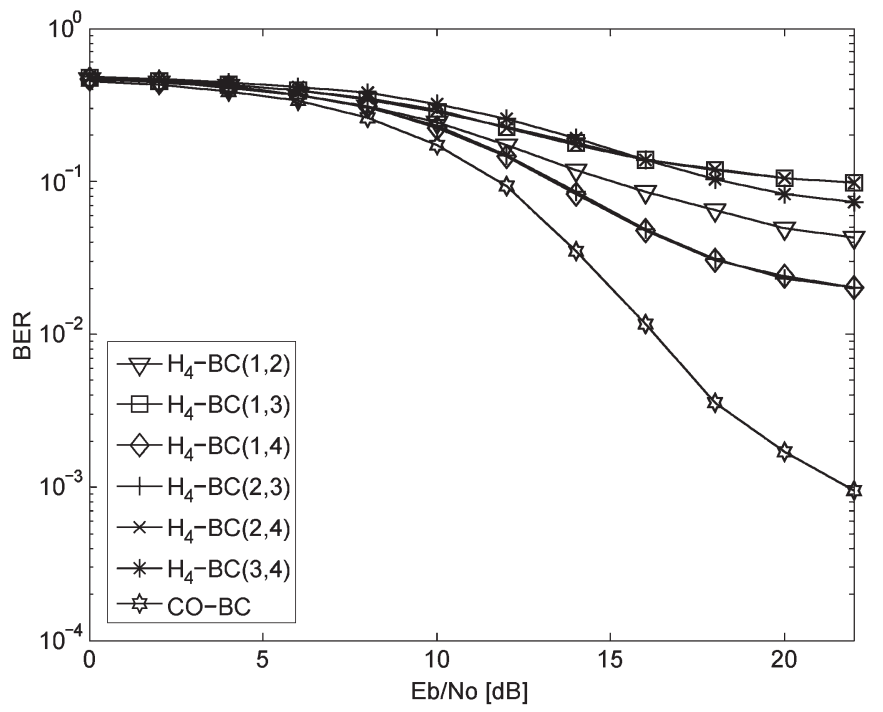

Fig. 6. Effects of the average CCG on the system performance of BC-CMSA in the presence of severe IFI and strong ISI, where $N_{f}=4$ and $T_{f}=5 \mathrm{~ns}$ are set so that $T_{f}<\tau_{R M S}=8.06 \mathrm{~ns}$ and $T_{s}<3 \tau_{R M S}$.

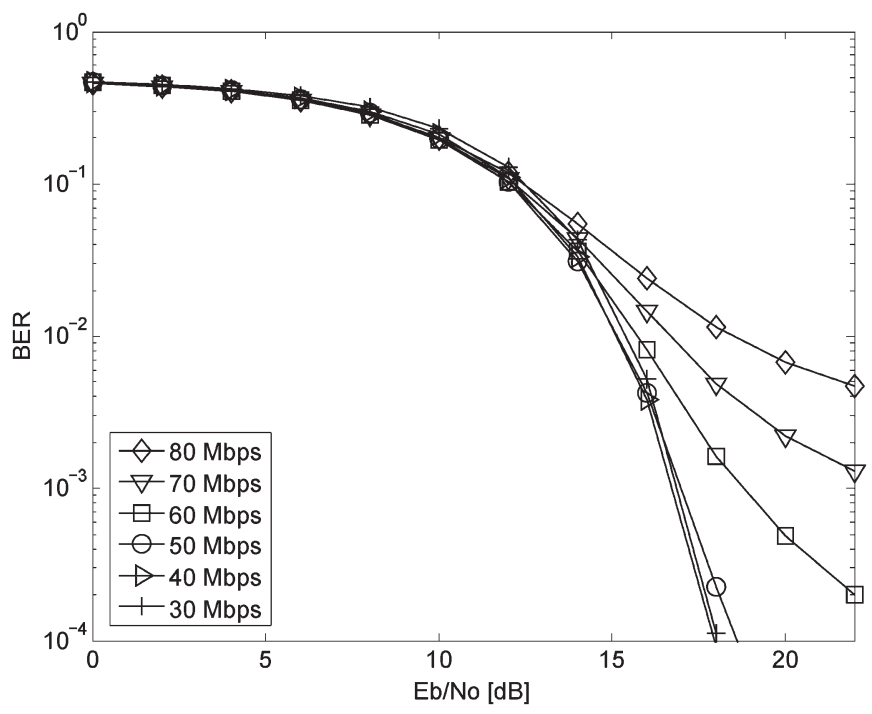

Fig. 7. BER performances comparison for CO-BC-CMSA with different data rates, where $N_{f}=2$ is fixed, and $T_{f}$ is set in accordance with the data rate.

Test Case 3: Higher data rate, which requires less $N_{f}$ or $T_{f}$, is desired. To better understand the effectiveness of the CO-BCbased premitigation with minimum $N_{f}=2$, the performances of the proposed CO-BC-CMSA with different data rates are illustrated in Fig. 7. The testing data rates are set from $30 \mathrm{Mb} / \mathrm{s}$ to $80 \mathrm{Mb} / \mathrm{s}$, with the frame length dropping from $16.7 \mathrm{~ns}$ to $6.25 \mathrm{~ns}$. With boosted data rates, IFI and ISI increase. It is shown that, for data rates of 30 and $40 \mathrm{Mb} / \mathrm{s}$, the performance degradation is invisible at $\mathrm{BER}=10^{-4}$, whereas the system with a data rate of $50 \mathrm{Mb} / \mathrm{s}$ performs a little worse than the system with a data rate of 30 or $40 \mathrm{Mb} / \mathrm{s}$. However, when the data rate is boosted beyond $50 \mathrm{Mb} / \mathrm{s}$, the system performance degradation becomes significant. The reason is that a frame length of less than $10 \mathrm{~ns}$ can no longer ensure the moderate IFI/ISI assumption. The signal components in the $p>2$ layers would contain significant power, and the effec-

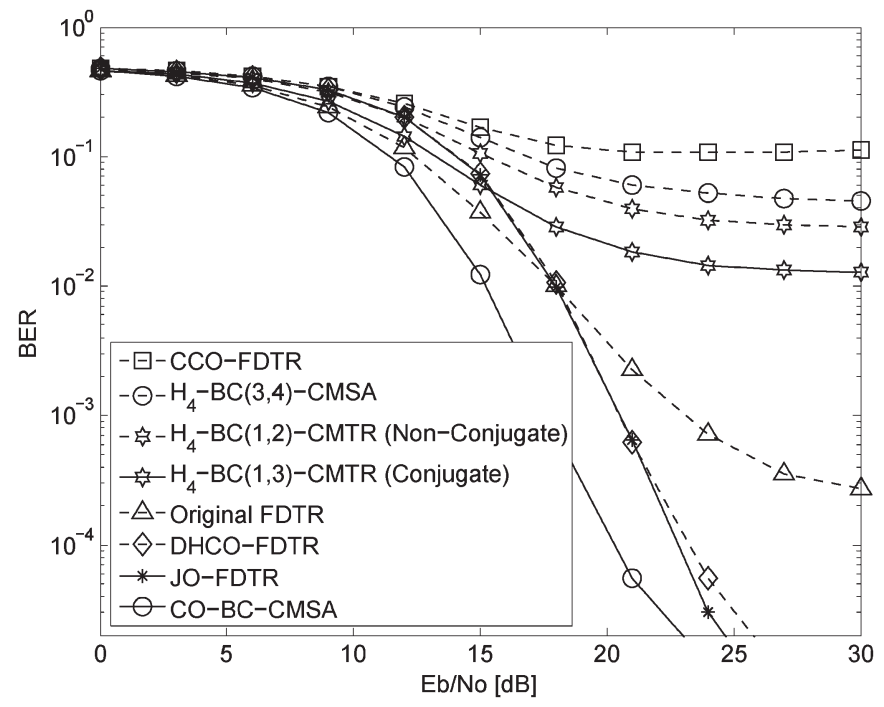

Fig. 8. Comparison of different IFI/ISI premitigation schemes between the BC-CMSA, FDTR, and CMTR systems. $N_{f}=4$ and $T_{f}=6.25$ ns are set for all schemes, whereas $T_{I}=T_{f}$ is used for BC-CMSA/CMTR, and $T_{I}=T_{s}$ is used for FDTR.

tive IFI/ISI would continue across multiple frames/symbols. In this scenario, premitigation alone is not so effective. The low-complexity mitigation scheme for strong IFI/ISI in the BC-CMSA system is an interesting topic for future work.

Test Case 4: In this test case, the proposed code optimization scheme is compared with [10] and [20] to offer more insights into the receiver-specific IFI/ISI premitigation schemes. For fair comparison, the frame number is set as $N_{f}=4$ with a frame duration of $T_{f}=6.25 \mathrm{~ns}$ for all schemes, and the integral duration is set as $T_{I}=T_{f}$ for the BC-CMSA and CMTR systems, whereas $T_{I}=T_{s}$ is used for the FDTR system, respectively. In particular, we consider the IFI-robust BC design for CMTR [10], which employs the so-called conjugate pair $\mathbf{H}_{4}(1,3)$ for transmission. In addition, the performance-boosting schemes for FDTR [20] is considered, which involves the delay-hopping code optimization (DHCO), chip code optimization (CCO), and the joint optimization (JO) of both chip and delay-hopping codes. For the relevant parameter setting with regard to [20], we choose the delay hopping code of period 4 as $[0,0,1,3]$ with a delay chip duration of $T_{c}=T_{\omega}$ and the orthogonal chip code of period 16 by cascading all the four row vectors of the Walsh-BC $\mathbf{H}_{4}$. As illustrated in Fig. 8, CO-BC-CMSA, CMTR with conjugate pair $\mathbf{H}_{4}(1,3)$, and JO-FDTR all show better robustness to IFI/ISI than their nonoptimized counterparts. In fact, for the AcR-based FDTR system, the IFI/ISI components are mainly cross correlations between different "leaked" partial channel responses. By noting the correlation properties of the UWB channel [24], [20] exploits the specific nature of the AcR, which enables the DHCO to suppress IFI/ISI. On the other hand, the IFI/ISI components in the ED-based BC-CMSA and CMTR are mainly autocorrelations of different "leaked" partial channel responses, which cannot effectively be suppressed by DHCO; therefore, the optimized BCs are evoked for IFI/ISI mitigation. Note that the chip code for FDTR is similar to the CO-BC for CMSA or the conjugate code pair used for CMTR, because they all change the polarities of pulses and can suppress 
some IFI/ISI component. However, both CCO-FDTR and the optimized CMTR with conjugate pair are not very effective in combating IFI/ISI, as illustrated in Fig. 8, because they exploit only ordinary orthogonal Walsh-BC $\mathbf{H}_{4}$, whose orthogonal property is not enough to ensure IFI/ISI-robust detection for these two systems. Contrary to the ordinary orthogonal BC, the proposed $\mathrm{CO}-\mathrm{BC}$ enjoys more favorable properties, i.e., shifted orthogonality and shifted repetition, which achieve effective IFI/ISI premitigation for the BC-CMAS system. Moreover, CO-BC-CMSA outperforms JO-FDTR and CMTR with conjugate pair in the practical $E_{b} / N_{0}$ region, although the nonoptimized $\mathbf{H}_{4}$-BC-CMSA performs worse than the nonoptimized FDTR and CMTR.

\section{CONCLUSiON}

In this paper, we have proposed a simple IFI/ISI premitigation scheme for the BC-CMSA system through a code optimization approach. We have analyzed the performance of the binary-modulated BC-CMSA system in the presence of moderate IFI/ISI to show the effectiveness of $\mathrm{BC}$ selection for performance enhancement. With the aim of maximizing the proposed performance metric, i.e., the average CCG, we have constructed the statistically optimal $\mathrm{BC}$ that enjoys the following two IFI/ISI-robust properties: 1) shifted orthogonality and 2) shifted repetition. The optimal BC tailors the IFI/ISI patterns to match the predetection operation and energy detection mechanism of the CMSA receiver; therefore, the leaked signal energy is partially utilized to enhance detection. Simulation results have demonstrated the effectiveness of the proposed IFI/ISI premitigation scheme.

\section{APPENDIX A}

\section{Channel-Averaged Statistics}

Taking the noise effect into account, (11) is extended as

$$
\begin{aligned}
\bar{\Lambda}\left[\mathbf{r}_{i}(t) \mid \mathbf{b}^{(m)}\right] & =\mathrm{E}_{h}\left\{\int_{0}^{T_{f}}\left(\mathbf{b}^{(m)} \mathbf{r}_{i}(t)\right)^{2} d t\right\} \\
& =\bar{S}_{i}^{(m)}+\bar{I}_{i, 1}^{(m)}+\bar{I}_{i, 2}^{(m)}+\bar{\xi}_{i, 1}^{(m)}+\bar{\xi}_{i, 2}^{(m)}
\end{aligned}
$$

where the interference-free signal component is

$$
\bar{S}_{i}^{(m)}=\mathrm{E}_{h}\left\{\left(c_{i, 0}^{(m)}\right)^{2}[\mathbf{R}]_{1,1}\right\}=\left(c_{i, 0}^{(m)}\right)^{2} \bar{\varepsilon}_{0} .
$$

The interference component from $p>0$ layers is

$$
\bar{I}_{i, 1}^{(m)}=\mathrm{E}_{h}\left\{\sum_{p^{\prime}=p=1}^{N_{f}-1}\left(c_{i, p}^{(m)}\right)^{2}[\mathbf{r}]_{p+1, p^{\prime}+1}\right\}=\sum_{p=1}^{N_{f}-1}\left(c_{i, p}^{(m)}\right)^{2} \bar{\varepsilon}_{p}
$$

and the interference component that is generated by the crosslayer correlations is

$$
\bar{I}_{i, 2}^{(m)}=\mathrm{E}_{h}\left\{\sum_{p=0}^{N_{f}-1} \sum_{p^{\prime}=0, p^{\prime} \neq p}^{N_{f}-1} c_{i, p}^{(m)} c_{i, p^{\prime}}^{(m)}[\mathbf{R}]_{p+1, p^{\prime}+1}\right\} \approx 0 .
$$

For the noise components,

$$
\bar{\xi}_{i, 1}^{(m)}=\mathrm{E}_{h}\left\{2 \int_{0}^{T_{f}} \mathbf{c}_{i}^{(m)} \mathbf{g}(t) \cdot n_{i}^{(m)}(t) d t\right\}
$$

is the signal-by-noise component, and finally, the noise-bynoise component is given as

$$
\bar{\xi}_{i, 2}^{(m)}=\mathrm{E}_{h}\left\{\int_{0}^{T_{f}}\left(n_{i}^{(m)}(t)\right)^{2} d t\right\}=\int_{0}^{T_{f}}\left(n_{i}^{(m)}(t)\right)^{2} d t .
$$

\section{APPENDIX B}

\section{STATISTIC ANALYSIS ON $\bar{X}_{i}\left(d^{\prime}\right)$}

Note that, in $\bar{X}_{i}\left(d^{\prime}\right)$, the signal component $\bar{X}_{i, S}\left(d^{\prime}\right)$ is irrelevant to noise; therefore, the mean and variance of $\bar{X}_{i}\left(d^{\prime}\right)$ are $\mathrm{E}_{n}\left\{\bar{X}_{i}\left(d^{\prime}\right)\right\}=\bar{X}_{i, S}\left(d^{\prime}\right)+\mathrm{E}_{n}\left\{\bar{X}_{i, \xi}\left(d^{\prime}\right)\right\}$ and $\operatorname{Var}_{n}\left\{\bar{X}_{i}\left(d^{\prime}\right)\right\}=$ $\operatorname{Var}_{n}\left\{\bar{X}_{i, \xi}\left(d^{\prime}\right)\right\}$, respectively.

We start by the derivation for $\mathrm{E}_{n}\left\{\bar{X}_{i}\left(d^{\prime}\right)\right\}$. Let us first derive the term $\bar{X}_{i, S}\left(d^{\prime}\right)$ as

$$
\begin{aligned}
\bar{X}_{i, S}\left(d^{\prime}\right) & =\bar{S}_{i}^{(m)}\left(d^{\prime}\right)-\bar{S}_{i}^{\left(m^{\prime}\right)}\left(d^{\prime}\right)+\bar{I}_{i, 1}^{(m)}\left(d^{\prime}\right)-\bar{I}_{i, 1}^{\left(m^{\prime}\right)}\left(d^{\prime}\right) \\
& \approx N_{f}^{2} \bar{\varepsilon}_{1}+\left[\left(c_{i, 1}^{(m)}\left(d^{\prime}\right)\right)^{2}-\left(c_{i, 1}^{\left(m^{\prime}\right)}\left(d^{\prime}\right)\right)^{2}\right] \bar{\varepsilon}_{2} \\
& \approx \lambda N_{f}^{2} \bar{\varepsilon}_{g}+\mu\left[\left(c_{i, 1}^{(m)}\left(d^{\prime}\right)\right)^{2}-\left(c_{i, 1}^{\left(m^{\prime}\right)}\left(d^{\prime}\right)\right)^{2}\right] \bar{\varepsilon}_{g}
\end{aligned}
$$

where the first approximation is based on the moderate IFI/ISI, i.e., $\bar{\varepsilon}_{0}+\bar{\varepsilon}_{1} \approx \bar{\varepsilon}_{g}=\sum_{p=0}^{N_{f}-1} \bar{\varepsilon}_{p} \gg \sum_{p=2}^{N_{f}-1} \bar{\varepsilon}_{p}$; therefore, only the $p=0,1$ layers are considered here for simplicity. The second approximation introduces $\lambda=\bar{\varepsilon}_{0} / \bar{\varepsilon}_{g}$ as the ratio between the averaged energy of $g_{0}(t)$ and $g(t)$, and $\mu=$ $1-\lambda$. In addition, the mean of $\bar{X}_{i, \xi}\left(d^{\prime}\right)$ is given in [22] as $\mathrm{E}_{n}\left\{\bar{X}_{i, \xi}\left(d^{\prime}\right)\right\} \approx 0$. By summarizing the results, the mean of $\bar{X}_{i}\left(d^{\prime}\right)$ is given as

$\mathrm{E}_{n}\left\{\bar{X}_{i}\left(d^{\prime}\right)\right\} \approx \lambda N_{f}^{2} \bar{\varepsilon}_{g}+\mu\left[\left(c_{i, 1}^{(m)}\left(d^{\prime}\right)\right)^{2}-\left(c_{i, 1}^{\left(m^{\prime}\right)}\left(d^{\prime}\right)\right)^{2}\right] \bar{\varepsilon}_{g}$.

We continue to derive $\operatorname{Var}_{n}\left\{\bar{X}_{i}\left(d^{\prime}\right)\right\}$. Following [22] and [6], $\bar{\xi}_{i, 1}^{(m)}\left(d^{\prime}\right), \bar{\xi}_{i, 1}^{\left(m^{\prime}\right)}\left(d^{\prime}\right)$, and $\left[\bar{\xi}_{i, 2}^{(m)}\left(d^{\prime}\right)-\bar{\xi}_{i, 2}^{\left(m^{\prime}\right)}\left(d^{\prime}\right)\right]$ can be considered approximately uncorrelated for simplicity; then, $\operatorname{Var}_{n}\left\{\bar{X}_{i, \xi}\left(d^{\prime}\right)\right\}$ is approximated as

$$
\begin{array}{r}
\operatorname{Var}_{n}\left\{\bar{X}_{i, \xi}\left(d^{\prime}\right)\right\} \approx \operatorname{Var}_{n}\left\{\bar{\xi}_{i, 1}^{(m)}\left(d^{\prime}\right)\right\}+\operatorname{Var}_{n}\left\{\bar{\xi}_{i, 1}^{\left(m^{\prime}\right)}\left(d^{\prime}\right)\right\} \\
+\operatorname{Var}_{n}\left\{\bar{\xi}_{i, 2}^{(m)}\left(d^{\prime}\right)-\bar{\xi}_{i, 2}^{\left(m^{\prime}\right)}\left(d^{\prime}\right)\right\}
\end{array}
$$

where the last term is the noise-by-noise component, and its variance is given in [22] as $\operatorname{Var}_{n}\left\{\xi_{i, 2}^{(m)}\left(d^{\prime}\right)-\xi_{i, 2}^{\left(m^{\prime}\right)}\left(d^{\prime}\right)\right\} \approx$ 
$2 N_{0}^{2} N_{f}^{2} T_{I} W$. In addition, the variance of $\bar{\xi}_{i, 1}^{(m)}\left(d^{\prime}\right)$ is derived as

$$
\begin{aligned}
& \operatorname{Var}_{n}\left\{\bar{\xi}_{i, 1}^{(m)}\left(d^{\prime}\right)\right\} \\
& =\mathrm{E}_{n}\left\{\mathrm{E}_{h}\left\{2 \int_{0}^{T_{f}} \mathbf{c}_{i}^{(m)}\left(d^{\prime}\right) \cdot \mathbf{g}(t) \cdot n_{i}^{(m)}(t) d t\right\}\right. \\
& \left.\times \mathrm{E}_{h}\left\{2 \int_{0}^{T_{f}} \mathbf{c}_{i}^{(m)}\left(d^{\prime}\right) \cdot \mathbf{g}(\tau) \cdot n_{i}^{(m)}(\tau) d \tau\right\}\right\} \\
& =2 N_{0} N_{f} \sum_{p=0}^{N_{f}-1}\left(c_{i, p}^{(m)}\left(d^{\prime}\right)\right)^{2} \bar{\varepsilon}_{p} \\
& \approx 2 N_{0} N_{f}\left(N_{f}^{2} \bar{\varepsilon}_{0}+\left(c_{i, p}^{(m)}\right)^{2} \bar{\varepsilon}_{1}\right) \\
& \approx 2 N_{0} N_{f}\left(\lambda N_{f}^{2}+\mu\left(c_{i, p}^{(m)}\right)^{2}\right) \bar{\varepsilon} g
\end{aligned}
$$

where the last two approximations are obtained with moderated IFI/ISI assumption, as shown in (24). Similarly, the variance of $\bar{\xi}_{i, 1}^{\left(m^{\prime}\right)}\left(d^{\prime}\right)$ is given as

$$
\operatorname{Var}_{n}\left\{\bar{\xi}_{i, 1}^{\left(m^{\prime}\right)}\left(d^{\prime}\right)\right\} \approx 2 N_{0} N_{f} \mu\left(c_{i, p}^{\left(m^{\prime}\right)}\right)^{2} \bar{\varepsilon}_{g} .
$$

By summarizing the results, the variance of $\bar{X}_{i}\left(d^{\prime}\right)$ is given as

$$
\begin{aligned}
\operatorname{Var}_{n}\{ & \left.\bar{X}_{i}\left(d^{\prime}\right)\right\} \approx 2 N_{0}^{2} N_{f}^{2} T_{i} W+2 N_{0} N_{f} \bar{\varepsilon}_{g} \\
& \times\left\{\lambda N_{f}^{2}+\mu\left[\left(c_{i, 1}^{(m)}\left(d^{\prime}\right)\right)^{2}+\left(c_{i, 1}^{\left(m^{\prime}\right)}\left(d^{\prime}\right)\right)^{2}\right]\right\} .
\end{aligned}
$$

\section{APPENDIX C \\ DERIVATION OF $\gamma_{i}\left(d^{\prime}\right)$ With High SNR AsSUMPTION}

Recall that SNR $:=E_{b} / N_{0}=N_{f} \bar{\varepsilon}_{g} / N_{0}$, and rewrite (16) as

$$
\gamma_{i}\left(d^{\prime}\right)=\frac{1}{m_{1}+m_{2}}
$$

where

$$
m_{1}=\frac{2}{\mathbf{S N R}} \times \frac{\lambda+\frac{\mu}{N_{f}^{2}}\left[\left(c_{i, 1}^{(m)}\left(d^{\prime}\right)\right)^{2}+\left(c_{i, 1}^{\left(m^{\prime}\right)}\left(d^{\prime}\right)\right)^{2}\right]}{\left\{\lambda+\frac{\mu}{N_{f}^{2}}\left[\left(c_{i, 1}^{(m)}\left(d^{\prime}\right)\right)^{2}-\left(c_{i, 1}^{\left(m^{\prime}\right)}\left(d^{\prime}\right)\right)^{2}\right]\right\}^{2}}
$$

$$
m_{2}=\frac{1}{\mathbf{S N R}^{2}} \times \frac{2 T_{i} W}{\left\{\lambda+\frac{\mu}{N_{f}^{2}}\left[\left(c_{i, 1}^{(m)}\left(d^{\prime}\right)\right)^{2}-\left(c_{i, 1}^{\left(m^{\prime}\right)}\left(d^{\prime}\right)\right)^{2}\right]\right\}^{2}}
$$

When $\mathbf{S N R}$ is high such that $2 / \mathbf{S N R} \gg 1 / \mathbf{S N R}^{2}$, which results in $m_{1} \gg m_{2}$, we have

$$
\gamma_{i}\left(d^{\prime}\right) \approx \frac{1}{m_{1}}=\frac{\mathbf{S N R}}{2} \beta_{i}\left(d^{\prime}\right)
$$

where the conditional CCG $\beta_{i}\left(d^{\prime}\right)$ is given in (19).

\section{APPENDIX D}

\section{PROOF OF LEMMA 2}

Proof: The $\mathrm{BC}$ of interest is $\widehat{\mathbf{B}}_{2 \times N_{f}}$, and without loss of generality, we first choose one of the codewords, e.g., $\mathbf{b}=$ $\left[b_{0}, \ldots, b_{N_{f}-1}\right]$, for study. In addition, consider the special case $d=\left(m_{i-1}=c, m_{i}=c\right)$ ( $c$ is a temporary codeword index of $\mathbf{b}$ ), and the one-frame shifted version of $\mathbf{b}$ is $\tilde{\mathbf{b}}_{i, 1}(c, c)=$ $\left[b_{N_{f}-1}, b_{0}, \ldots, b_{N_{f}-2}\right]$. Then, the correlation between the two codewords is

$$
\mathbf{b} \tilde{\mathbf{b}}_{i, 1}^{T}(c, c)=\sum_{j=0}^{N_{f}-1} b_{j} b_{\bmod _{N_{f}}[j-1]} .
$$

Because $b_{j}= \pm 1$ and $b_{j} b_{\bmod _{N_{f}}[j-1]}= \pm 1, \forall j \in\left[0, N_{f}-1\right]$ (in this proof, $j$ is confined in $\left[0, N_{f}-1\right]$ ), (34) is bounded as

$$
-N_{f} \leq \sum_{j=0}^{N_{f}-1} b_{j} b_{\bmod _{N_{f}}[j-1]} \leq N_{f} .
$$

The BC with shifted repetition can achieve this bound, and two cases are involved as follows.

1) The equation in the right-hand side of (35) holds only when $b_{j} b_{\bmod _{N_{f}}[j-1]}=1, \forall j$. Correspondingly

$$
b_{j}=b_{\bmod _{N_{f}}[j-1]}= \pm 1, \quad \forall j .
$$

Then, two candidates $\mathbf{b}^{(c 1)}=[1, \ldots, 1]$ with $\left[\mathbf{b}^{(c 1)}\right]_{j}=$ 1 or $\mathbf{b}^{(c 2)}=[-1, \ldots,-1]$ with $\left[\mathbf{b}^{(c 2)}\right]_{j}=-1$ are obtained.

2) The equation in the left-hand side of (35) holds only when $b_{j} b_{\bmod N_{f}}[j-1]=-1, \forall j$. Correspondingly

$$
\left\{\begin{array} { c } 
{ b _ { j } = 1 } \\
{ b _ { \operatorname { m o d } N _ { f } } [ j - 1 ] = - 1 }
\end{array} \text { or } \left\{\begin{array}{c}
b_{j}=-1 \\
b_{\bmod _{N_{f}}}[j-2]=1
\end{array} \quad \forall j .\right.\right.
$$

Then, two candidates $\mathbf{b}^{(c 3)}=[1,-1, \ldots, 1,-1]$ with $\left[\mathbf{b}^{(c 3)}\right]_{j}=(-1)^{j+1}$ or $\mathbf{b}^{(c 4)}=[-1,1, \ldots,-1,1]$ with $\left[\mathbf{b}^{(c 4)}\right]_{j}=(-1)^{j}$ are obtained.

With the partial shifted-repetition constraint (only the interference pattern $d=(c, c)$ is considered), only four possible candidates $\left\{\mathbf{b}^{(c 1)}, \mathbf{b}^{(c 2)}, \mathbf{b}^{(c 3)}, \mathbf{b}^{(c 4)}\right\}$ are reserved to construct the desired $\mathrm{BC}$. Then, it is easy to verify with other interference patterns, i.e., $d=\left(c, c^{\prime}\right), c \neq c^{\prime}$, that there are two $\mathrm{BCs}$ that meet the constraints of shifted repetition and shifted repetition. The two BCs are $\widehat{\mathbf{B}}_{2 \times N_{f}}^{(1)}$, which consists of $\mathbf{b}^{(c 1)}$ and $\mathbf{b}^{(c 4)}$, and $\widehat{\mathbf{B}}_{2 \times N_{f}}^{(2)}$, which consists of $\mathbf{b}^{(c 2)}$ and $\mathbf{b}^{(c 3)}$. In addition, we notice that, the two BCs only have a sign difference, i.e., $\widehat{\mathbf{B}}_{2 \times N_{f}}^{(1)}=-\widehat{\mathbf{B}}_{2 \times N_{f}}^{(2)}$, and the sign does not affect 
code properties. For notional simplicity, we choose $\widehat{\mathbf{B}}_{2 \times N_{f}}=$ $\widehat{\mathbf{B}}_{2 \times N_{f}}^{(1)}$ as the exclusive representative. Therefore, $\widehat{\mathbf{B}}_{2 \times N_{f}}=$ $\left[\widehat{\mathbf{B}}_{2 \times 2}, \widehat{\mathbf{B}}_{2 \times 2}, \ldots, \widehat{\mathbf{B}}_{2 \times 2}\right]$ with $\widehat{\mathbf{B}}_{2 \times 2}=\left[\begin{array}{cc}1 & 1 \\ -1 & 1\end{array}\right]$ is the only binary antipodal code with shifted orthogonality and shifted repetition, ignoring the sign difference.

\section{REFERENCES}

[1] L. Yang and G. Giannakis, "Ultrawideband communications: An idea whose time has come," IEEE Signal Process. Mag., vol. 21, no. 6, pp. 26-54, Nov. 2004.

[2] M. Win and R. Scholtz, "Ultrawide bandwidth time-hopping spreadspectrum impulse radio for wireless multiple-access communications," IEEE Trans. Commun., vol. 48, no. 4, pp. 679-689, Apr. 2000.

[3] M. Win and R. Scholtz, "On the energy capture of ultrawidebandwidth signals in dense multipath environments," IEEE Commun. Lett., vol. 2, no. 9, pp. 245-247, Sep. 1998.

[4] R. Hoctor and H. Tomlinson, "Delay-hopped transmitted-reference RF communications," in Proc. IEEE Conf. UWBST, 2002, pp. 265-269.

[5] M. Ho, V. Somayazulu, J. Foerster, and S. Roy, "A differential detector for an ultrawideband communications system," in Proc. IEEE VTC, Spring 2002, pp. $1896-1900$.

[6] J. Choi and W. Stark, "Performance of ultrawideband communications with suboptimal receivers in multipath channels," IEEE J. Sel. Areas Commun., vol. 20, no. 9, pp. 1754-1766, Dec. 2002.

[7] K. Witrisal, G. Leus, M. Pausini, and C. Krall, "Equivalent system model and equalization of differential impulse radio UWB systems," IEEE J. Sel. Areas Commun., vol. 23, no. 9, pp. 1851-1862, Sep. 2005.

[8] X. Dong, L. Jin, and P. Orlik, "A new transmitted reference pulse cluster system for UWB communications," IEEE Trans. Veh. Technol., vol. 57, no. 5, pp. 3217-3224, Sep. 2008.

[9] D. Goeckel and Q. Zhang, "Slightly frequency-shifted reference ultrawideband UWB radio," IEEE Trans. Commun., vol. 55, no. 3, pp. 508-519, Mar. 2007.

[10] A. D'amico and U. Mengali, "Code-multiplexed UWB transmittedreference radio," IEEE Trans. Commun., vol. 56, no. 12, pp. 2125-2132, Dec. 2008.

[11] Y. Ying, M. Ghogho, and A. Swami, "Block-coded modulation and noncoherent detection for impulse radio UWB," IEEE Signal Process. Lett., vol. 15 , pp. 112-115, 2008.

[12] A. Molisch, J. Foerster, and M. Pendergrass, "Channel models for ultrawideband personal area networks," IEEE Trans. Wireless Commun., vol. 10, no. 6, pp. 14-21, Dec. 2003.

[13] K. Witrisal, G. Leus, G. Janssen, M. Pausini, F. Troesch, T. Zasowski, and J. Romme, "Noncoherent ultrawideband systems," IEEE Signal Process. Mag., vol. 26, no. 4, pp. 48-66, Jul. 2009.

[14] J. Proakis and M. Salehi, Digital Communications. New York: McGrawHill, 1995

[15] M. Sahin and H. Arslan, "Intersymbol interference in high-data-rate UWB communications using energy detector receivers," in Proc. IEEE ICU, 2005, pp. 176-179.

[16] C. Krall, K. Witrisal, G. Leus, and H. Koeppl, "Minimum mean-squareerror equalization for second-order Volterra systems," IEEE Trans. Signal Process., vol. 56, no. 10, pp. 4729-4737, Oct. 2008.

[17] T. Zasowski, F. Troesch, and A. Wittneben, "Partial channel-state information and intersymbol interference in low-complexity UWB PPM detection," in Proc. IEEE ICUWB, 2006, pp. 369-374.

[18] V. Lottici, L. Wu, and Z. Tian, "Intersymbol interference mitigation in high-data-rate UWB systems," in Proc. IEEE ICC, 2007, pp. 4299-4304.

[19] Z. Tian and G. Giannakis, "A GLRT approach to data-aided timing acquisition in UWB radios-Part I: Algorithms," IEEE Trans. Wireless Commun., vol. 4, no. 6, pp. 2956-2967, Nov. 2005.

[20] M. Pausini, G. Janssen, and K. Witrisal, "Performance enhancement of differential UWB autocorrelation receivers under ISI," IEEE J. Sel. Areas Commun., vol. 24, no. 4, pp. 815-821, Apr. 2006.

[21] H. Gao, T. Lv, X. Su, and B. Liu, "Optimized block-coded noncoherent UWB impulse radio with IFI and ISI premitigation," in Proc. IEEE ICC, May 2010, pp. 1-5.

[22] D. Mu, Z. Qiu, and X. Zhang, "Performance analysis of UWB noncoherent receiver with block-coded modulation scheme," IEEE Commun. Lett., vol. 14, no. 6, pp. 509-511, Jun. 2010.
[23] J. Foerster, Channel Modeling Subcommittee Final Rep. (doc.: IEEE802-15-02/490rl-SG3a). IEEE P802. 15 Working Group for Wireless Personal Area Networks (WPANs), Feb. 2002.

[24] K. Witrisal and M. Pausini, "Statistical analysis of UWB channel correlation functions," IEEE Trans. Veh. Technol., vol. 57, no. 3, pp. 1359-1373, May 2008.

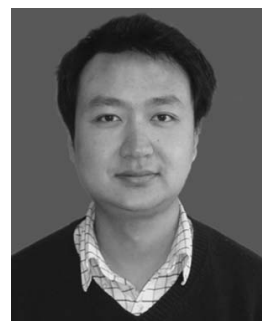

Hui Gao (S'10) received the B.Eng. degree in 2007 from Beijing University of Posts and Telecommunications, Beijing, China, where is currently working toward the Ph.D. degree with the School of Information and Communication Engineering.

Since 2009, he also served as a Research Assistant for the Wireless and Mobile Communications Technology R\&D Center, Tsinghua University, Beijing. His research interests include ultrawideband wireless communications, physical-layer network coding, and multiuser multiple-input-multiple-output systems.

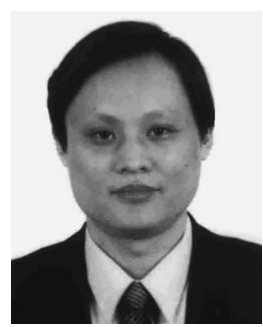

Xin Su (M'02) received the M.S. and Ph.D. degrees in electronic engineering from the University of Electronic Science and Technology of China, Chengdu, China, in July 1996 and December 1999, respectively

From January 2000 to October 2001, he was a Research Fellow with the Department of Electronic Engineering, Tsinghua University, Beijing, China, where he worked on software radio theory and technology and applications for personal communications. Since November 2001, he has been with the School of Information Science and Technology, Tsinghua University, Beijing, where he is currently a Professor with the Research Institute of Information Technology and the Vice Director of the Wireless and Mobile Communications Technology R\&D Center. As a Faculty Member and Graduate Supervisor, he has been teaching courses in wireless and mobile communications. His research interests include wireless and mobile networks, broadband wireless access, software-defined radio, and wireless multimedia communications.

Dr. Su is the Chairman of the International Mobile TelecommunicationsAdvanced (IMT-Advanced) Technology Working Group, Ministry of Industry and Information Technology of the People's Republic of China, and the Vice Chairman of the Innovative Wireless Technology Working Group, China Communications Standards Association.

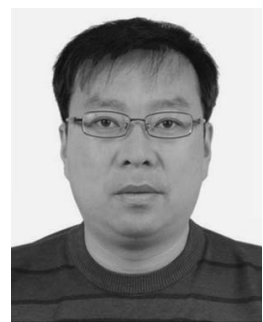

Tiejun Lv (M'08) received the M.S. and Ph.D. degrees in electronic engineering from the University of Electronic Science and Technology of China, Chengdu, China, in 1997 and 2000, respectively.

From January 2001 to December 2002, he was a Postdoctoral Fellow with Tsinghua University, Beijing, China. From September 2008 to March 2009, he was a Visiting Professor with the Department of Electrical Engineering, Stanford University, Stanford, CA. He is currently a Professor with the School of Information and Communication Engineering, Beijing University of Posts and Telecommunications. He has published more than 100 technical papers on the physical layer of wireless mobile communications.

Dr. Lv is a Senior Member of the Chinese Electronics Association. He received the "Program for New Century Excellent Talents in University" Award from the Ministry of Education, China, in 2006. 


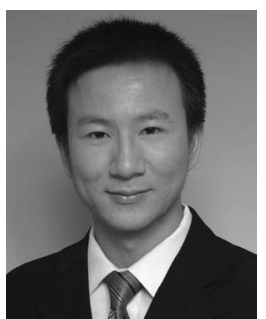

Shaoshi Yang (S'09) received the B.Eng. degree in information engineering from Beijing University of Posts and Telecommunications, Beijing, China, in 2006. He is currently working toward the Ph.D. degree in wireless communications with the School of Electronics and Computer Science, University of Southampton, Southampton, U.K., through scholarships from both the University of Southampton and the China Scholarship Council.

From November 2008 to February 2009, he was an Intern Research Fellow with the Communications Technology Laboratory, Intel Labs China, Beijing, where he focused on Channel Quality Indicator Channel design for mobile WiMAX (802.16 m). His research interests include multiuser dection/multiple-input-mutliple-output detection, multicell joint/distributed processing, and interference management.

Mr. Yang is a Junior Member of the Isaac Newton Institute for Mathematical Sciences, Cambridge, U.K., and a Technical Program Committee Member of the 23rd Annual IEEE International Symposium on Personal, Indoor, and Mobile Radio Communications (PIMRC 2012).

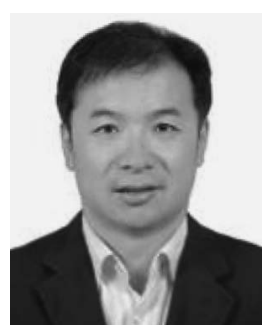

Yueming Lu received the B.S. and M.S. degrees of computer science from Xian University of Architecture and Technology, Xian, China, in 1994 and 1997, respectively, and $\mathrm{Ph} . \mathrm{D}$. degree of computer architecture from Xian Jiaotong University, Xian, in 2000 .

From 2000 to 2003, he was a Researcher with the Optical Network Group Pacific, Lucent. In 2003, he joined Beijing University of Posts and Telecommunications, Beijing, China, where he is currently a Professor with the Key Laboratory of Trustworthy Distributed Computing and Service, Ministry of Education. His research interests include routing protocols, network design, and distributed computing.

Dr. Lu is a Member of the Chinese Computer Federation. 